\title{
Mathematical Models for Expansive Growth of Cells with Walls
}

\author{
J.K.E. Ortega *, S.W.J. Welch \\ Bioengineering Laboratory, Department of Mechanical Engineering, University of Colorado \\ Denver, Colorado 80217-3364 USA
}

\begin{abstract}
Plants, algae, and fungi are essential for nearly all life on earth. Through photosynthesis, plants and algae convert solar energy to chemical energy in the form of organic compounds that sustains essentially all life on earth. In addition, plants and algae convert the carbon dioxide produced by respiring organisms to oxygen that is needed for respiration. Fungi decompose complex organic compounds produced by respiring organisms so that molecules can be recycled in photosynthesis and respiration. Plants, algae, and fungi have one important feature in common, their cells have walls. Expansive growth and its regulation are central to the life and development of plant, algal, and fungal cells, i.e. cells with walls. In recent decades there has been an explosion of information relevant to expansive growth of cells with walls. Mathematical models have been constructed in an attempt to organize and evaluate this information, to gain insight, to evaluate hypotheses, and to assist in the selection and development of new experimental studies. In this article some of the mathematical models constructed to study expansive growth of cells with walls are reviewed. It is nearly impossible to review all relevant research conducted in this area. Instead, the review focuses on the development of mathematical equations that have been used to model expansive growth, morphogenesis, and growth rate regulation of cells with walls. Also, relevant experimental findings are reviewed, conceptual models are presented, and suggestions for future research are proposed. The authors have attempted to provide an overview that is accessible to researchers that are not working in this field.
\end{abstract}

Keywords and phrases: plants, algae, fungi, growth models, growth equations, augmented growth equations

Mathematics Subject Classification: 97M10, 97M60, 92C80

\section{Introduction}

Expansive growth and its regulation are fundamental to development, morphogenesis, and environmental responses of plant, algal and fungal cells. Prior research demonstrates the need for mathematical models

${ }^{*}$ Corresponding author. E-mail: joseph.ortega@ucdenver.edu 
that describe expansive growth, morphogenesis, and growth rate regulation of plant, algal and fungal cells, e.g. [14-16,24,27-29, 35, 38,45,46,63,73]. Importantly, expansive growth of plant, algal, and fungal cells (cells with walls) employ the same physical principles. The cell transports or produces active solutes inside the plasma membrane and absorbs water from its surroundings through the process of osmosis. The absorption of water produces turgor pressure that stresses the wall. The wall is biochemically loosened, reducing both wall stresses (stress relaxation) and turgor pressure (turgor pressure relaxation). More water is absorbed in response to the decrease in turgor pressure, extending (deforming) the loosened wall. The wall deformation produces an increase in wall surface area (expansion) and a thinner wall. New wall polymers and other wall materials are added to maintain a nearly constant wall thickness. The series of events (i.e. wall loosening, wall stress relaxation, turgor pressure relaxation, water uptake, wall expansion, an increase in turgor pressure, an increase in wall stresses, and wall loosening again) is repeated continually during expansive growth.

The wall deformation behavior during expansive growth depends on the wall's biochemically-mediated mechanical properties, but both irreversible and reversible (elastic) wall deformations occur simultaneously. Mathematically, a constitutive equation relates wall deformation (strain) behavior to wall stresses produced by the turgor pressure. The pressure probe is an instrument that can directly measure and control the magnitude of the turgor pressure in single plant, algal, and fungal cells. Pressure probe experiments demonstrate that controlling the magnitude of the turgor pressure and/or the wall mechanical properties can regulate expansive growth rate, e.g. $[14,15,47,49,55]$, however biologically controlled modification the wall mechanical properties is the predominate method employed to regulate morphogenesis, e.g. $[4,28]$.

Most plant and algal cells exhibit "diffuse" growth, where the entire wall chamber undergoes irreversible and elastic deformation during expansive growth $[49,55]$. Two examples of cells that exhibit diffuse growth are cells in plant organs (e.g. stems, roots, and leaves) and algal internode cells. However, some plant cells (e.g. pollen tubes and root hairs), fungal hyphae, and some fungal sporangiophores exhibit "tip" growth [30] where only a portion of the wall at the apical tip (in a growth zone) undergoes both irreversible and elastic deformation during expansive growth $[49,55]$. Some sporangiophores exhibit "intercalary" growth where only the wall within the growth zone (located immediately below a spherical sporangium) undergoes both irreversible and elastic deformation during expansive growth [49,55]. Examples of tip growth and intercalary growth can be found in the development of the sporangiophores of Phycomyces blakesleeanus. The sporangiophores undergo five stages of development (stages I, II, III, IV, and V) where stage IV is further divided into three sub-stages (IVa, IVb, and IVc) $[10,13,55]$. Stage I exhibits tip growth and stage IV exhibits intercalary growth.

To some extent the mechanical properties of the cell wall depends on the structure and architecture of the wall, e.g. $[4,9,10,16,65,67,68,73]$. The general composition of plant and algal walls can be described as cellulose microfibrils embedded in an amorphous matrix composed predominately of hemicelluloses and pectins, with small amounts of structural proteins [18]. Similarly, the general composition of the fungal wall can be described as microfibrils (composed of chitin and $\beta$-glucans) embedded in an amorphous matrix composed predominately of chitosan, glycoproteins, and lipids $[13,30,70]$. The wall polymers in plant, algal, and fungal cells are linked together by covalent bonds, hydrogen bonds, hydrophobic interaction, and ionic associations [70].

The microfibrils are extruded to the inner wall surface by protein-based molecular machinery embedded in the plasma membrane. In plant and algal cells, cellulose synthase complexes extrude cellulose microfibrils to the inner wall [18]. In fungal cells, chitin synthetases extrude chitin microfibrils to the inner wall $[13,30,70]$. All the wall materials destined to be microfibrils and matrix polymers are transported by vesicles to the plasma membrane and released to the inner wall via exocytosis $[3,6,77]$. 
From a structural and architectural perspective, the microfibrils are cross-linked by matrix polymers. Experimental evidence suggests that molecular agents that affect load-bearing cross-links between microfibrils are used to regulate wall mechanical properties and deformation behavior $[17,18]$. Breaking the cross links allows the microfibrils to separate [39] and produces a controlled polymer creep often referred to as "wall loosening". The wall loosening produces wall stress relaxation and turgor pressure relaxation that in turn produces water uptake, wall deformation, and an increase in cell volume.

Two wall-loosening mechanisms that have been extensively studied are thought to affect the hemicellulose network and the pectin network that cross-link cellulose microfibrils. Expansins are pH-dependent wall-loosening proteins that catalyze wall stress relaxation and wall extension directly, but are not enzymes $[17,18]$. The mechanism by which expansins produce wall loosening is unknown, but experimental and theoretical evidence $[17,18,76]$ indicate that expansins may affect hydrogen bonds within the hemicellulose network that cross-link cellulose microfibrils. Expansins are thought to be a primary wall-loosening agent for cells in organs of higher plants (e.g. stems, roots and leaves).

Calcium mediated cross-links of matrix polymers' carboxyl groups within the pectin network are also thought to be involved in wall loosening $[7,57,62]$. The carboxyl groups of newly synthesized pectin is mostly methyl esterified which prevents calcium cross-links from forming between pectin polymers. Subsequently an enzyme (pectin methylesterases) removes the methyl group allowing the carboxyl groups to form cross-links between pectin polymers. It is hypothesized that new pectin delivered to the inner wall via exocytosis, is subsequently de-esterified and loosens the wall by competing for and removing calcium from load-bearing cross-links. Breaking and making calcium-mediated pectin cross-links are thought to be a wall-loosening mechanism used by some algal cells [7,62] and pollen tubes [57].

Experimental investigations have identified many physical, mechanical, biological, and chemical processes that are involved in expansive growth of cells with walls. However, the sequence, interrelationship, and contribution of these processes to any growth event cannot be determined by experimental results alone. Generally, a growing amount of experimental results and information at the macroscopic and microscopic levels has generated a need to develop mathematical models to organize and relate these many processes both qualitatively and quantitatively.

Early mathematical models for expansive growth of cells with walls were derived from relevant underlying physical principles. Because all processes must obey the laws of physics, modelling the underlying physical principles assures that the obtained growth behavior is physically possible. Early models recognized the importance of interrelated physical and biological processes in growth and growth regulation of cells with walls. Thus, these early models were constructed by developing equations describing physical principles that are implicitly dependent on relevant biological processes, i.e. biophysical equations.

\section{Global biophysical models for expansive growth rate}

\subsection{Growth equations}

Lockhart [38] derived the first set of physically based mathematical equations that model expansive growth of cells with walls. The biophysical equations model two underlying physical processes of expansive growth, water uptake and wall deformation,

$$
\underbrace{\frac{1}{V}\left(\frac{d V_{w}}{d t}\right)}_{\text {rate of change in water volume }}=\underbrace{L(\Delta \Pi-P)}_{\text {rate of water uptake }} .
$$

Equation (2.1) describes a relationship between the relative rate of change in water volume within the cell, $\left(d V_{w} / d t\right) / V$, and the relative rate of water uptake. Equation (2.1) is written in relative terms, i.e. 
the terms are divided by the magnitude of the volume, $V ; t$ is the time, $L$ is the relative membrane hydraulic conductance $\left(L=L_{p} A / V\right), L_{p}$ is the membrane hydraulic conductivity, $A$ is the membrane area, $\Delta \Pi$ is the osmotic pressure difference across the membrane (the solute reflection coefficient is assumed to be unity and omitted from these equations), $P$ is the turgor pressure (a gauge pressure relative to atmospheric pressure as measured with a pressure probe, $P_{I}-P_{\text {atm }}$, where $P_{I}$ is the pressure inside the plasma membrane and $P_{\text {atm }}$ is defined to be zero).

$$
\underbrace{\frac{1}{V}\left(\frac{d V_{c w c}}{d t}\right)}_{\text {rate of change in cell wall chamber volume }}=\underbrace{\phi\left(P-P_{c}\right)}_{\text {irreversible deformation rate }} .
$$

Equation (2.2) describes the relative rate of change in volume of the cell wall chamber, $\left(d V_{c w c} / d t\right) / V$, as equal to the relative irreversible deformation rate of the wall. The biophysical variable, $\phi$, is the relative irreversible extensibility of the wall and $P_{C}$ is the critical turgor pressure which is related to the yield threshold, $Y$. Because the rates of change in volume of the cell wall chamber and water are approximately equal, $\left.\left(d V_{c w c} / d t\right) / V \approx\left(d V_{w} / d t\right) / V\right)$, Eq. (2.1) may be substituted into Eq. (2.2) to obtain an expression for the equilibrium pressure, $P_{e q}$,

$$
P_{e q}=\frac{L \Delta \Pi+\phi P_{C}}{\phi+L} .
$$

Equations (2.1), (2.2) and (2.3) are termed the Growth Equations [73] and provide a mathematical model for the growth rate of cells with walls. The two underlying physical processes of water uptake rate (Eq. (2.1)) and irreversible wall deformation rate (Eq. (2.2)) are interdependent during growth and the presence of $P$ in each of the equations couples them to reflect this condition.

Equation (2.2) is a global equation that can be viewed as a biophysical constitutive equation for the cell wall. Equation (2.2) has shown utility in modeling experimental results because the expansive growth rate, $\left(d V_{c w c} / d t\right) / V$, changes when $\phi$ and $P$ change in magnitude (when $P>P_{C}$ ). The underlying local constitutive equation for Eq. (2.2) is that of a Bingham fluid [5]. Researchers have used Eq. (2.2) to explore the behavior of expansive growth rate after changing the turgor pressure and/or mechanical properties of the wall e.g. [27, 29, 53, 56,73]. Other researchers have employed Eq. (2.2) in global or local form as the constitutive equation for the cell wall to investigate multi-dimensional expansive growth (morphogenesis) e.g. [19,60], growth rate regulation (during pollen tube oscillatory growth e.g. [34] and sporangiophore development e.g. [56]), and the molecular mechanism employed by plant cells to regulate mechanical properties of the wall e.g. [59].

\subsection{Augmented Growth Equations.}

Subsequently, Ortega [45,51] augmented the Growth Equations with additional terms (Augmented Growth Equations). Equation (2.1) was augmented with a transpiration term that accounts for the water loss to the environment from cells with walls that are directly exposed to the atmosphere [51],

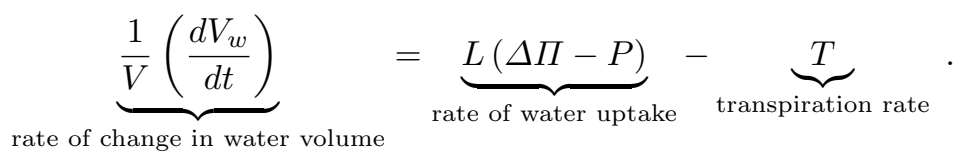

Equation (2.4) describes the relative rate of change in water volume, $\left(d V_{w} / d t\right) / V$, of the cell as the difference between the rate of water uptake and transpiration rate [46-48,51]. Where $T$ is the relative rate of change in water volume lost via transpiration (relative volumetric transpiration rate), i.e. $T=$ $\left(d V_{T} / d t\right) / V$. Equation (2.4) has shown utility for fungal sporangiophores because the water uptake occurs 
near the base of the long cylindrical cell and water is lost through transpiration from the stalk exposed to the atmosphere. Pressure probe methods employing Equation (2.4) have been developed to study transpiration in sporangiophores. There is good agreement between the predicted and measured turgor pressure behavior e.g. $[47,51]$. In the case when the cell is not exposed to the atmosphere, then $T=0$ and Equation (2.1) is recovered.

Equation (2.2) was augmented with a term that accounts for elastic deformation within the growing wall $[14,45]$,

$$
\underbrace{\frac{1}{V\left(\frac{d V_{c w c}}{d t}\right)}}_{\text {rate of change in cell wall chamber volume }}=\underbrace{\phi\left(P-P_{c}\right)}_{\text {irreversible deformation rate }}+\underbrace{\frac{1}{\varepsilon} \frac{d P}{d t}}_{\text {elastic deformation rate }} .
$$

Equation (2.5) describes the relative rate of change in volume of the cell wall chamber, $\left(d V_{c w c} / d t\right) / V$, as the sum of irreversible deformation rate and reversible (elastic) deformation rate of the wall [4548]. The biophysical variable $\varepsilon$ is the volumetric elastic modulus. In the case when turgor pressure does not change, then $d P / d t=0$ and Equation (2.2) is recovered. The underlying local constitutive equation for Equation (2.5) is that of a Maxwell-Bingham viscoelastic model [45]. Because the relative rate of change in volume of the cell contents (mostly water) and cell wall chamber are approximately equal, a biophysical equation describing the rate of change of turgor pressure is obtained by combining Equation (2.4) and Equation (2.5) with the elimination of $\left(d V_{w} / d t\right) / V$ and $\left(d V_{c w c} / d t\right) / V$, because $\left(d V_{w} / d t\right) / V \approx\left(d V_{c w c} / d t\right) / V[47,48]$

$$
\underbrace{\left(\frac{d P}{d t}\right)}_{\text {rate of change of turgor pressure }}=\varepsilon[\underbrace{L(\Delta \Pi-P)}_{\text {rate of water uptake }}-\underbrace{\phi\left(P-P_{c}\right)}_{\text {irreversible deformation rate }}-\underbrace{T}_{\text {transpiration rate }}] .
$$

Equation (2.6) states that the rate of change of turgor pressure is proportional to the difference in magnitudes of water uptake rate and the irreversible deformation rate of the wall and volumetric transpiration rate. Equation (2.6) may be solved to determine the turgor pressure change after an instantaneous change in the magnitude of the one or more of the inclusive biophysical variables and/or the relative volumetric transpiration rate [48],

$$
P(t)=\left(P_{0}-P_{n e q}\right) \exp [-\varepsilon(\phi+L) t]+P_{n e q},
$$

where

$$
P_{n e q}=\frac{L \Delta \Pi+\phi P_{c}-T}{\phi+L} .
$$

Equation (2.7) describes an exponential change in turgor pressure from $P_{0}$ (turgor pressure at $t=0$ ) to $P_{n e q}$ (new equilibrium turgor pressure). The exponential decay has a time constant, $t_{c}=[\varepsilon(\phi+L)]^{-1}$. The expression for $P_{n e q}$ (Eq. (2.8)) describes the equilibrium turgor pressure for a growing cell that is transpiring [48]. Interestingly, Eq. (2.8) establishes a relationship between the equilibrium turgor pressure and the transpiration rate. It can be seen that an increase or decrease in transpiration rate will decrease or increase the new equilibrium turgor pressure, respectively. In the special case where there is not any transpiration, Eq. (2.8) reduces to Eq. (2.3).

In the past these global biophysical equations have been adapted to study wall deformation in one direction (unidirectional growth or elongation growth) $[27,29,47,53,55,56,63]$. Sometimes it is mistakenly thought that these unidirectional equations only model unidirectional stress on the cell wall, thus producing wall deformation behavior and measured magnitudes of inclusive biophysical variables that are not accurate for actual cells experiencing multi-directional wall stresses and multi-directional wall 
deformation. This is not true. The turgor pressure is the driving force for the wall deformation in these global biophysical equations and the turgor pressure produces the same multi-directional wall stresses experienced by the wall in vivo. Furthermore, the resulting wall deformation is also multi-directional, however only one direction of the wall deformation is explicitly described and measured, i.e. only one component of the resulting multi-directional deformation is described and measured. So these biophysical equations implicitly account for multi-directional wall stresses and wall deformations produced by turgor pressure, thus the wall deformation (deformation rate and elongation rate) and obtained magnitudes of the inclusive global biophysical variables in the Augmented Growth Equations are accurate for the direction measured.

To illustrate, consider a cylindrical cell wall $10 \mathrm{~mm}$ in length and $150 \mu \mathrm{m}$ in diameter with a turgor pressure of $0.3 \mathrm{MPa}$ (these values represents the dimensions and turgor pressure of a typical nongrowing stage III sporangiophore of $P$. blakesleeanus). Increasing the turgor pressure by $0.1 \mathrm{MPa}$ with a pressure probe expands the wall $15 \mu \mathrm{m}$ in length and $2 \mu \mathrm{m}$ in radius (again typical values obtained from a stage III sporangiophore). The stage III sporangiophore does not grow and the wall deformations are elastic. The longitudinal component of the elastic modulus, $\varepsilon_{L}$, can be calculated using Eq. (2.5) modified for elongation growth and shown in Fig. 2 (panels B and C), $\varepsilon_{L}=\left(L_{i} / \Delta L\right) \Delta P=$ $(10 \mathrm{~mm} / 0.015 \mathrm{~mm})(0.1 \mathrm{MPa})=66.7 \mathrm{MPa}$, where $L$ is the length of the cell wall and the subscript " $i$ " refers to "initial". Similarly, the radial component of the elastic modulus, $\varepsilon_{R}$, can be calculated with the equation, $\varepsilon_{R}=\left(A_{i} / \Delta A\right) \Delta P=\left[R_{i}^{2} /\left(R_{f}^{2}-R_{i}^{2}\right)\right] \Delta P=\left(5625 \mu \mathrm{m}^{2} / 304 \mu \mathrm{m}^{2}\right)(0.1 \mathrm{MPa})=1.85 \mathrm{MPa}$, where $A$ is the cross-sectional area and the subscript " $f$ " refers to "final". The reciprocal of the global volumetric modulus for the whole cell wall may be obtained from the sum of the directional reciprocals, $1 / \varepsilon=1 / \varepsilon_{L}+1 / \varepsilon_{R}$, or the equation, $\varepsilon=\left(\varepsilon_{L} \varepsilon_{R}\right) /\left(\varepsilon_{L}+\varepsilon_{R}\right)=1.8 \mathrm{MPa}$. It is noted that the magnitude of $\varepsilon$ calculated from its directional components $\left(\varepsilon_{L}\right.$ and $\left.\varepsilon_{R}\right)$ is the same as the magnitude obtained from the cell wall volume, $V$, and change in cell wall volume, $\Delta V$, due to a change in turgor pressure, $\varepsilon=\left(V_{i} / \Delta V\right) \Delta P=18.0(0.1 \mathrm{MPa})=1.8 \mathrm{MPa}$. Importantly, additional information concerning the anisotropic mechanical behavior of the wall is obtained when the directional components $\left(\varepsilon_{L}\right.$ and $\left.\varepsilon_{R}\right)$ are determined. This anisotropic information may be used to guide and help validate constitutive relationships used in mathematical models.

\subsection{In Vivo Stress Relaxation and In Vivo Creep.}

In material science, the mechanical behavior and constitutive relationship for complicated composite materials similar to cell walls are often determined by stress relaxation and creep tests. In a stress relaxation test, a constant deformation (strain) is applied and the resulting load (stress) is measured as a function of time. In a creep test, a constant load (stress) is applied and the resulting deformation (strain) is measured as a function of time. The creep test and stress relaxation test were adapted to investigate the mechanical behavior of cell walls and to determine the constitutive equation for cell walls in living and growing cells, i.e. in vivo stress relaxation and in vivo creep experiments.

In vivo stress relaxation experiments are conducted by eliminating water uptake and transpiration from growing cells and measuring the turgor pressure as a function of time, $P(t)$, with the pressure probe $[14,15,53]$. In vivo stress relaxation experiments have been conducted on individual cells [53] and cells in plant tissue $[14,15]$. The time dependent turgor pressure, $P(t)$, is shown during stress relaxation of cells within the growth zone of pea stems in Fig. 1 from [14]. The turgor pressure of individual cells are measured with the pressure probe. The results demonstrate an exponential decay in $P$ to a constant value, $P_{C}$.

Importantly, the Growth Equations (Eqs. (2.1), (2.2) and (2.3)) cannot model this turgor pressure behavior. On the other hand, good agreement is obtained between the experimental results and the 


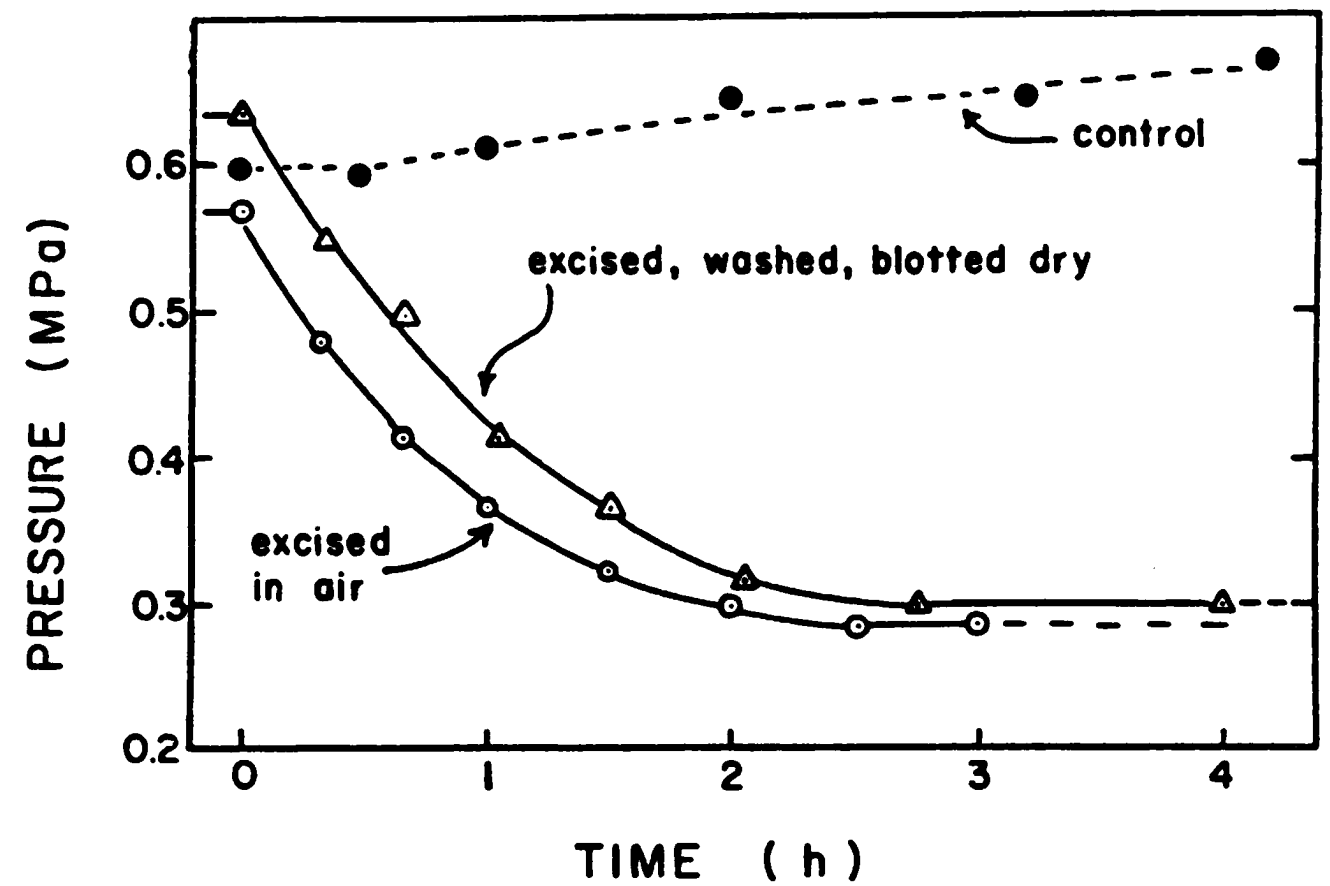

FiguRE 1. Stress relaxation of plant cells in the growth zone of pea stems. See text for description. Reproduced from Cosgrove (1985) [14], copyrighted by the American Society of Plant Biologists and is reprinted with permission.

predicted behavior for an in vivo stress relaxation experiment using the Augmented Growth Equations (Eqs. (2.4), (2.5) and (2.6)). Inspection of Eq. (2.6) shows that when both water uptake and transpiration are eliminated during an in vivo stress relaxation experiment, Eq. (2.6) becomes

$$
\frac{d P}{d t}=-\varepsilon \phi\left(P-P_{C}\right) \text {. }
$$

The solution for $P(t)$ is given by

$$
P(t)=\left(P_{i}-P_{C}\right) \exp (-\varepsilon \phi t)+P_{C} .
$$

The solution describes an exponential decay of the turgor pressure from some initial value, $P_{i}$, to the critical turgor pressure, $P_{C}$. The exponential decay has a time constant of $t_{c}=(\varepsilon \phi)^{-1}$.

Many walled cells exhibit expansive growth predominately by elongating, e.g. cells in elongating plant tissue (stems and roots), pollen tubes, root hairs, internode algal cells, fungal hyphae, and fungal sporangiophores. In vivo creep experiments are conducted on elongating single cells by producing a step-up or step-down in $P\left(P_{0} \pm \Delta P\right)$ with a pressure probe and measuring the resulting elongation as a function of time, $L(t)[53,56,63,64]$.

In vivo creep experiments conducted on internode cells of Chara corallina is shown in Fig. 2 from [63]. Fig. $2 \mathrm{~A}$ is the $P$-trace from the pressure probe $(P$ vs. $t)$ and shows a step-down in $P$ followed by a step-up in $P$. The elongation as a function of time, $L(t)$, is shown in Fig. 2 B, where the thin wiggly line beneath the darker lines is the measured $L(t)$. It can be seen that a sharp decrease and increase in $L$ occurs simultaneous to the $P$ step-down and $P$ step-up, respectively (elastic contraction and expansion of the wall). Also it is noted that the slope of the curve $(d L / d t$ or elongation rate) decreases after the 
$P$ step-down and increases after the $P$ step-up. If the sharp decrease and increase in $L$ after the $P$ step-down and after the $P$ step-up, respectively, (shown in Fig. $2 \mathrm{C}$ ) are removed from the $L(t)$ curve in Fig. $2 \mathrm{~B}$, then the $L(t)$ curve in Fig. $1 \mathrm{D}$ is produced.

Again, the Growth Equations cannot model the elongation behavior shown in Fig. 2 B. Equation (2.2) does model the decrease and increase in elongation rate obtained after the decrease and increase in $P$, but it cannot model the sharp and immediate changes in length (elastic changes) that occur with step changes in $P$ (Figs. 2 B and 2 C). In contrast, the Augmented Growth Equation (Eq. (2.5)) modified for elongation growth rate $(d L / d t)$ and shown at the top of panel $\mathrm{B}$, can accurately model the experimental results shown in Fig. 2 B. The dark straight lines on top of the thin wiggly curve are the $L(t)$ obtained with the Augmented Growth Equation. It can be seen that there is good agreement between experimental and modeled behavior of $L(t)$.

These results demonstrate that the Augmented Growth Equations can accurately model the results of in vivo stress relaxation and in vivo creep experiments. Importantly, good agreement has been demonstrated between theoretical results obtained from the Augmented Growth Equations and experimental results obtained from growing plant cells in pea stems $[14,15]$, fungal sporangiophores $[47,49,51,53,56]$, and algal internode cells $[63,64]$. In addition the Augmented Growth Equations have been modified to model expansive growth and water uptake of plant cells embedded in tissue and organs, where pressures within the apoplasm (cell walls and xylem) are frequently different from atmospheric pressure [48]. These modified Augmented Growth Equations for cells in tissue predict growth-induced water potentials [41,44] and can model growth behavior obtained experimentally [74]. Ortega [47,49] and Geitmann and Ortega [24] have reviewed these biophysical equations.

\subsection{Overview.}

The Growth Equations and Augmented Growth Equations are sets of biophysical equations that implicitly account for multi-directional wall stresses produced by turgor pressure and multi-directional wall deformations that produce a change in cell volume. Importantly, both sets of biophysical equations (Eqs. (2.1)-(2.3) and Eqs. (2.4)-(2.6)) model water uptake and wall expansion, and each set of equations are coupled by turgor pressure, thus explicitly demonstrating that they are interrelated processes. This is important because modelling expansive growth requires modelling both the processes of wall uptake and wall deformation (expansion).

The global constitutive relationship of the growing wall is revealed by the results of in vivo stress relaxation and in vivo creep experiments conducted with a pressure probe (Figs. 1 and 2). The experimental results indicate that both irreversible and elastic deformations occur in growing cell walls. In the Growth Equations, the global constitutive equation for the cell wall (Eq. (2.2)) cannot model the experimental results presented in Figs. 1 and 2. In contrast, in the Augmented Growth Equations, the global constitutive equation for the cell wall (Eq. (2.5)) can and does model the experimental results presented in Figs. 1 and 2. The predicted behaviors of the Augmented Growth Equations have been validated by many different pressure-probe experiments conducted on plant cells in tissue [14,15,42,43], algal cells $[63,64]$, and fungal cells $[47,49,51,53,55,56]$. Different pressure-probe experiments have been conducted to measure the magnitude and behavior of inclusive biophysical variables within the Augmented Growth Equations during development [56], with growth mutants [55], and during environmental responses [52]. Also, the Augmented Growth Equations have been extended to study whole plants growth behavior over long periods of time [36] and the affect of wall loosening agents on growth rate behavior [61].

In general these biophysical equations have demonstrated utility in understanding the relationship between expansive growth rate (and its regulation), mechanical properties and behavior of the wall, and turgor pressure magnitude and behavior. However, these biophysical equations have inherited limitations 


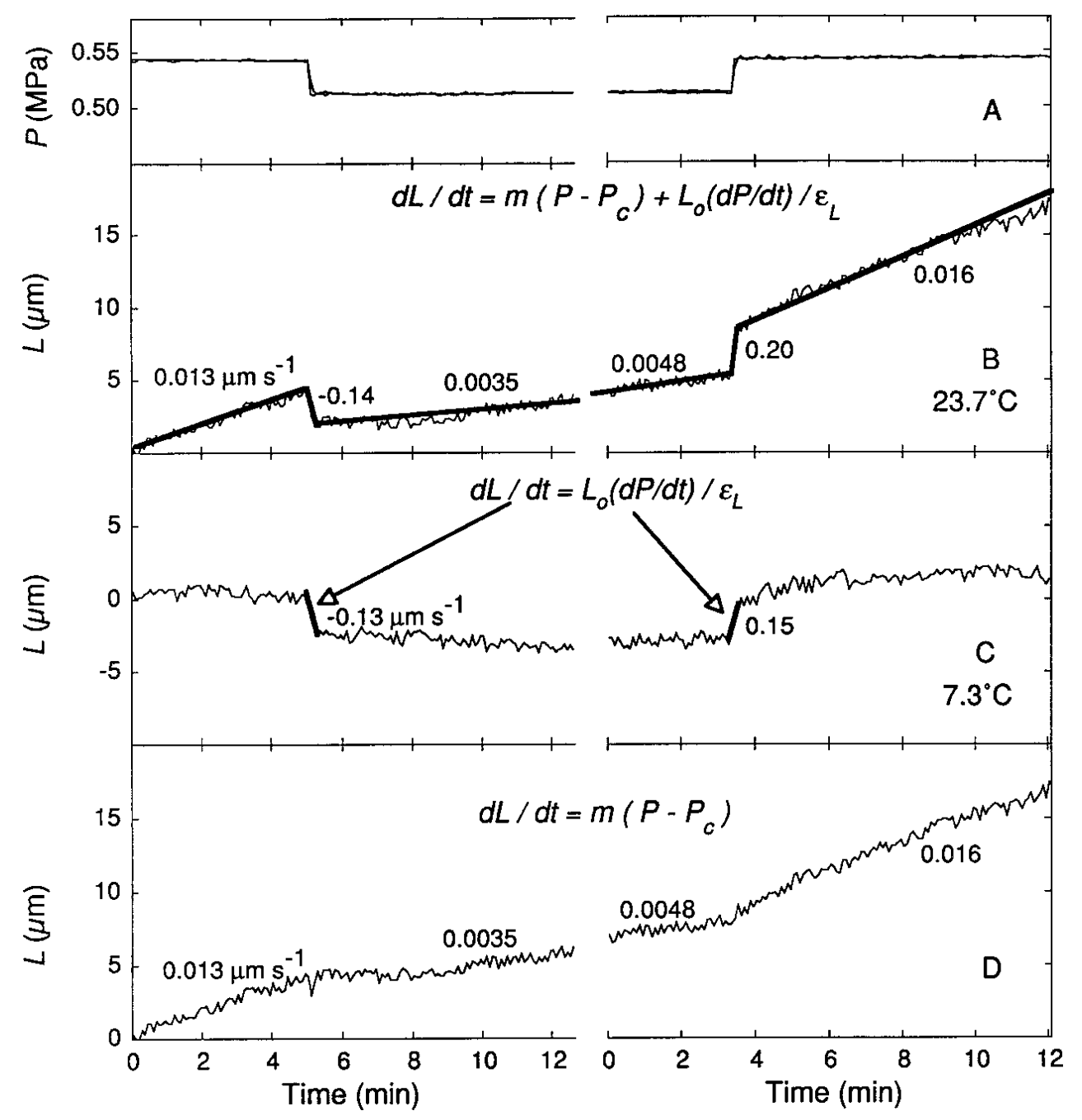

FiguRE 2. An in-vivo creep experiment conducted on an internode cell of $C$. corallina. (A) $P$-trace obtained from the pressure probe showing a step-down and step-up in $P$. (B) Elongation behavior at room temperature, $23.7^{\circ} \mathrm{C}$. (C) Elongation behavior of the same internode cell at colder temperature, $7.3^{\circ} \mathrm{C}$. (D) Elongation behavior after subtracting the elastic contraction and extension shown in (C). Reproduced from Proseus et al. (1999) [63], copyrighted by the American Society of Plant Biologists and is reprinted with permission.

because they are global. They cannot model local spatial or temporal changes in the wall that can enhance our understanding of morphogenesis and of the molecular mechanism of wall loosening. Other examples of shortcomings are that they do not explicitly model wall thinning rate, wall deposition rate, or the relationship between wall structure, wall chemistry, and mechanical properties.

There are a growing number of models that are constructed to address some of these shortcomings. In general, expansive growth of cells with walls involves multidimensional wall mechanics and multidimensional deformation behavior coupled with relevant biological processes. Modelling of the relationship between multidimensional growth behavior, multidimensional macroscopic mechanical properties, and 
the molecular mechanisms associated with their regulation (wall deposition, wall assembly, wall loosening, and wall stiffening) represents a challenging multi-scale modeling problem. A complete model for expansive growth requires a system model that couple the relevant biological processes with concomitant wall mechanical property modifications. Because the relevant biological processes and molecular mechanisms are yet to be completely understood, it is most useful if the models are constructed and developed to provide physical and biological insight into expansive growth that can guide the development of new experimental investigations that identify and explore relevant biological processes and mechanisms.

\section{Models for multidimensional wall deformation}

Experimental evidence indicates that the shape of cells with walls is obtained by producing anisotropic mechanical deformation of the wall, e.g. $[4,28,73]$. Some experimental evidence indicates a relationship between the anisotropic mechanical properties of the wall and the orientation of microfibrils embedded within the wall matrix, e.g. $[4,10,65,67,68,73]$. However, at least for plant cells, there is also evidence indicating that morphogenesis is more complicated and may also depend on dynamic processes involving the deposition rates of new wall polymers at specific locations, and the action of biologically active molecules, ions, proteins, and enzymes within the wall, e.g. [4,18]. In general, it is thought that anisotropic mechanical deformation is achieved by temporal and spatial regulation of wall mechanical properties that in turn is achieved by regulating the processes of wall deposition, wall assembly, wall loosening, and wall stiffening. It follows that mathematical models for morphogenesis require mathematical constitutive equations for multidimensional wall deformations.

\subsection{Irreversible wall deformations.}

Some models for multidimensional wall deformation employ constitutive equations exhibiting flow of material that are typically considered to be viscous fluids, Bingham fluids, or rate dependent viscoplastic solids. The stress is considered to be a function of the velocity gradient tensor, or equivalently the rate of strain tensor. Models that exclusively employ these constitutive equations do not capture the load bearing elastic stresses. The mathematical description of multidimensional wall deformation is a challenging problem and the mathematical models are still early in development. A review of some more recent models illustrates diverse approaches to solving this problem. Pietruszka [60] has proposed a three-dimensional constitutive model that simplifies to Eq. (2.2) in the one-dimensional case, thus it may be considered a three-dimensional extension of the Bingham fluid model. It is emphasized that the proposed three-dimensional model is local whereas Eq. (2.2) is global. However, the expansive growth and irreversible wall deformation are driven directly by the effective turgor pressure $(P-Y)$, instead of wall stresses generated by turgor pressure. Using the global effective pressure, instead of local stresses, to drive three dimensional wall expansion limits this model because the spatial distribution of stresses cannot be modeled. The model may be written as

$$
\frac{d u_{i j}}{d t}=u_{i k} \Phi_{k j}(P-Y)
$$

where $u$ is the strain tensor, $\Phi$ is the extensibility tensor, $P$ the scalar pressure and $Y$ the scalar pressure threshold. $u$ and $\Phi$ may be functions of position and time making the model local and allowing for modeling of experimentally observed changes in the wall extensibility. An advantage of this model is that for prescribed pressure Eq. (3.1) is a coupled system of linear ordinary differential equations that may easily be solved for the strains. For the case of homogeneous $u$ and $\Phi$ the model reduces to Eq. (2.2) with time dependent extensibility. A disadvantage is that elastic deformations are neglected, so the model does not include the physics demonstrated in Figs. 1 and 2. Solutions for this case are presented including 
a model for expansin activity that captures the time dependence of the extensibility by modeling the amount of expansin with a simple rate equation. The approach to modeling expansins activity presented in this work may be the basis for future efforts in this area.

Dumais et al. [19] developed a model for plant cell morphogenesis in tip growing cells that attempts to address the integration of two major processes, the deposition of new wall polymers at the inner wall surface and the anisotropic mechanical properties of the wall that may arise from the orientation of cellulose microfibrils within the wall matrix. They modeled the cell wall as a thin, transversely isotropic, viscoplastic membrane using an anisotropic multidimensional generalization of Bingham's equation. The wall thickness is invariant and the stresses and kinematic variables are considered constant through the wall thickness. The formulation models the tip as a dome with normal direction $n$, meridional direction $s$ and circumferential direction $\theta$ with corresponding Von-Mises yielding criterion

$$
\sigma_{e}=\left[A\left(\sigma_{s}-\sigma_{\theta}\right)^{2}+B\left(\sigma_{\theta}-\sigma_{n}\right)^{2}+C\left(\sigma_{n}-\sigma_{s}\right)^{2}\right]^{1 / 2} \geq \sigma_{y}
$$

where $A, B$ and $C$ are chosen to ensure that in the case of uniaxial stress the yielding criterion agrees with the one-dimensional Von-Mises yielding criterion. Their constitutive model uses the distortional energy as $H=\sigma_{e}^{2} / 2$ and the viscoplastic strain rates $\dot{\varepsilon}_{k}$ are assumed to be proportional to the overstress $\sigma_{e}-\sigma_{y}$ multiplied by the direction of greatest energy dissipation given by the gradient of the distortion energy.

$$
\dot{\varepsilon}_{k}=\Phi\left(\sigma_{e}-\sigma_{y}\right) \frac{1}{\|\nabla H\|} \frac{\partial H}{\partial \sigma_{k}} .
$$

The coefficient of proportionality $\Phi$ is the wall extensibility. Mechanical equilibrium relates the meridional and circumferential wall stresses to the pressure and principal curvatures

$$
\sigma_{s}=\frac{P}{2 \delta \kappa_{\theta}}, \quad \sigma_{\theta}=\frac{P}{2 \delta \kappa_{\theta}}\left(2-\frac{\kappa_{s}}{\kappa_{\theta}}\right),
$$

where $\kappa_{\theta}$ and $\kappa_{s}$ are the principal curvatures and $P$ is the turgor pressure. The curvatures change throughout the dome thus the distribution of stresses is not uniform in the dome even though the pressure is uniform along the cell wall. Material velocities are related to the strain rates and the resulting system of ordinary differential equation may be integrated numerically to obtain the evolving tip shape. The deformations considered are not infinitesimal but given that elastic contributions to the deformations were not included they did not need to consider the nonlinearities associated with elastic-plastic deformations.

The wall deposition is not explicitly modeled, but is implicitly accounted for by simply maintaining a constant wall thickness. Also, the wall deformation anisotropy is not modeled using a microfibril model but instead using a flow coupling parameter to control the ratio of yield stresses in coordinate directions. Through the use of a flow rule the flow coupling parameter was related to the ratio of strain rates in the coordinate directions. Thus, the spatial distribution of anisotropic mechanical properties and relevant biological processes are not explicitly modeled. Also, the system of equations describing expansive growth and morphogenesis constitute a feedback loop, which makes it difficult to predict cell shape from the flow coupling alone. This model can generate many of the shapes found during growth and development of cells with walls, even the formation of the spherical sporangium of fungal sporangiophores and beaded elongation growth exhibited by some tip-growing cells, e.g. pollen tubes. Given the dependence of stress on strain rates, and not strains, this model is not directed towards capturing the physics associated with stress relaxation and creep experiments previously described.

Campas and Mahadevan [8] present an axisymmetric thin shell model for tip-growing cells. They consider the cell wall to be a viscous fluid with a non-homogeneous viscosity to model the spatially 
varying enzymatic regulation of cross-links. Material addition (wall deposition) is included with a nonhomogeneous mass source in the apical region that models the fusion of secretory vesicles with the plasma membrane. Their model includes a set of coupled algebra and differential equations representing mechanical equilibrium, a Newtonian fluid constitutive equation, and a wall deposition model. An analysis of their model indicates that a single dimensionless parameter explains the observed variability in shapes found in tip-growing cells by accounting for the relative roles of cell wall assembly and expansion. The dimensionless parameter is $a / R_{0}$, where $a$ characterizes the spatial variation of secretion of new wall polymers and $R_{0}$ is a characteristic radius of curvature. This model is able to reproduce shapes in morphological studies that are observed in tip-growing cells. Also, this model asymptotically captures the distribution of wall deformation behavior as it transforms from viscous fluid behavior at and near the apex to elastic behavior (represented by high viscosity) in the cylindrical region removed from the apex by assuming a cylindrical shape. Included in this work is a description of the multiscale modeling issues that arise in describing the expansive growth of cell walls.

Dyson and Jensen [20] apply the model of Green and Friedman [26] that considers the wall to be a thin axisymmetric membrane of viscous fluid reinforced by embedded cellulose microfibrils. Their model did not include elastic deformations or stresses in the matrix material (the fluid membrane) but they did include infinitesimal elastic stretching of the microfibrils. Also their model included a microfibril model that allows consideration of the effect of changing microfibril orientation within the wall and across the wall thickness. The evolution equation for the microfibril direction vector a (derived in Green and Friedman $[26])$ is

$$
\frac{\partial a}{\partial t}+(U \cdot \nabla) a+(a \cdot e \cdot a) a=(a \cdot \nabla) U,
$$

where $U$ is the material velocity vector and $e$ is the rate of strain tensor defined as

$$
e=\frac{1}{2}\left(\nabla U+\nabla U^{T}\right) .
$$

Defining a fibril source term $G(x, t)$ the evolution equation for the microfibril density $\rho$ is

$$
\frac{\partial \rho}{\partial t}+\nabla \cdot(\rho U)=G(x, t) .
$$

Defining a mass source term $F(x, t)$ the viscous sheet must satisfy conservation of mass

$$
\nabla \cdot U=F(x, t)
$$

and conservation of momentum

$$
\nabla \cdot \sigma=0,
$$

where the Cauchy stress is related to the fiber orientation and the strain rate by the constitutive model

$$
\sigma_{i j}=-P \delta_{i j}+2 \mu_{0} e_{i j}+\mu_{1} a_{i} a_{j}+\mu_{2} a_{i} a_{j} a_{k} a_{l} e_{k l}+2 \mu_{3}\left(a_{i} a_{l} e_{j l}+a_{j} a_{m} e_{m i}\right) .
$$

Here $\mu_{0}$ is the usual viscosity for a Newtonian fluid and $\mu_{2}, \mu_{3}$ may be interpreted as viscosities associated with the presence of the embedded fibers. The term $\mu_{1} a_{i} a_{j}$ does not depend on the strain rate and it is interpreted as tension in the fiber direction. This term is not an elastic term as the fiber length does not change thus the magnitude of the stress in the fiber does not change but the fiber does reorient thus changing the directions that the stresses are acting. Although cell shapes were not reproduced with this model nor was it validated by comparison to experimental data, it may represent an important addition to the literature with respect to the spatial distribution and mechanical effects of microfibril orientation given the novel approach to tracking the microfiber orientation and distribution. 
Dyson et. al. [21] consider the dynamics of the hemicellulose cross-links between the stiff cellulose microfibrils in the wall. They treat the cell wall as an incompressible fluid with a prescribed extensional flow field and examine the strain-enhanced breakage of the cross-links as well as the enzyme-mediated cross-link kinetics. The microfibrils are oriented circumferentially and the cross-links are aligned perpendicular to the microfibrils in the longitudinal direction (the direction of cell wall deformation). The microfibrils and cross-links are deposited at the inner wall and are transported towards the outer wall by the flow field. The flow field is such that as the cross-links move to the outer wall as they are stretched in the longitudinal direction resulting in a non-uniform longitudinal extensional stress in the radial direction with the maximum extensional stress being close to the outer cell wall. As the cross-links approach the outer cell wall the model assumes they fail contributing to longitudinal growth through yielding. The authors also consider enzyme-induced decreases in the amount of cross-link extension before failure. In their model the enzymatic activity is uniform throughout the cell wall. They are able to produce quantitative results consistent with Eq. (2.2) indicating that their microscale description of cross-link kinetics will help advance the understanding of plant cell wall growth. The authors of this work make the pertinent observation that microscale models of the type presented here will be useful for multiscale models such as that of Huang et al. [32] (reviewed below). The model proposed by Ortega [49] is similar to this model in some respects. Both models conclude that the cross-links bear more of the load as they move towards the outer cell wall. The model proposed by Ortega [49] differs in that the induced wall loosening activity is high at the inner wall surface and decreases through the cell wall thickness towards the outer cell wall; see Section 5 of this review.

\subsection{Irreversible and Elastic Wall Deformations.}

The models described above generally employ a constitutive equation in which the stress depends on the rate of strain but not on the strain itself, effectively neglecting the load bearing capacity of the wall's elasticity. While the models described above are capable of producing wall shapes observed in cells with walls, they cannot be used to completely describe the mechanics in the wall. Importantly, the effective constitutive equations for each of the models described above cannot reproduce the experimentally results obtained from an in vivo stress relaxation experiment (Fig. 1) and an in vivo creep experiment (Fig. 2). A complete description of the wall mechanics must include the wall elasticity and in many cases address the difficulties associated with finite deformations.

Finite deformation constitutive models that include elastic deformation as well as irreversible deformation and that are similar to models of elastic-plastic deformation in engineering material science have recently received attention. A fundamental kinematic quantity appearing in finite deformation mechanics is the deformation gradient, which is a mapping of the undeformed configuration to the deformed configuration and is defined for a material particle as

$$
\mathbf{F}=\frac{\partial \boldsymbol{x}}{\partial \boldsymbol{X}}
$$

where $\boldsymbol{x}$ is coordinate of a material point in the deformed configuration and $\boldsymbol{X}$ is the coordinate of the same material particle in the reference (undeformed) configuration. Such models utilize a decomposition of the deformation gradient suggested by Rodriguez et al. [66] as a product of an elastic component with an irreversible (growth) component.

$$
\mathbf{F}=\mathbf{F}^{G} \mathbf{F}^{E}
$$

This mapping may be interpreted as growth represented by a mapping, $\mathbf{F}^{G}$, from the reference configuration to an intermediate stress-free configuration followed by a mapping, $\mathbf{F}^{E}$, to the final configuration. One of the attractive features of this decomposition is that it facilitates representation of residual stresses 
commonly found in growing tissue. The use of this kinematic mapping is not without criticism due to the fact that the intermediate configuration is not continuous (although the final configuration is) and the question as to what are the proper reference configurations to use for newly added material is an open question [2]. The first criticism also applies to elastic-plastic modeling of engineering materials using the same decomposition of the deformation gradient but with $\mathbf{F}^{G}$ replace by $\mathbf{F}^{P}$, where $\mathbf{F}^{P}$ represents plastic deformation. The second criticism is an added complexity faced by analysts who are considering material addition. The elastic component of the overall deformation is usually modeled as a hyperelastic material with constitutive equation

$$
\mathbf{S}=2 \frac{\partial \Psi}{\partial \mathbf{C}^{E}}
$$

where $\mathbf{S}$ is the second Piola-Kirchoff stress, $\Psi$ is the elastic strain energy and $\mathbf{C}^{E}$ is the right Cauchy-Green strain tensor defined as

$$
\mathbf{C}^{E}=\left(\mathbf{F}^{E}\right)^{T} \mathbf{F}^{E} \text {. }
$$

The second Piola-Kirchoff stress and the Cauchy (physical) stress are related by

$$
\sigma=J^{-1} \mathbf{F}^{E} \mathbf{S}\left(\mathbf{F}^{E}\right)^{T}
$$

where $J=\operatorname{det}\left(\mathbf{F}^{E}\right)$. The mechanical equilibrium equation is

$$
\nabla \cdot \sigma=0
$$

where the derivatives are taken with respect to the deformed configuration. The analyst wishing to use this formulation must specify the elastic constitutive equation(s), i.e. the strain energy, for the elastic components of the material. A constitutive model for the irreversible (growth) component of the deformation is also required. A major difficulty with this formulation is that unlike small displacement formulations the coordinates of material particles in the deformed configuration are not known a-priori and must be found through an iterative process typically requiring the full machinery of the finite element method for large deformations. Given the mathematical difficulties associated with this formulation, analytic solutions for boundary value problems where the loads are prescribed and the deformations and stresses are found through solution of the model equations do not exist. It is possible to specify the elastic and growth components of the deformation and then determine the resulting stresses and required loads using the constitutive and equilibrium equations. This inverse approach is a standard method to examining the behavior of constitutive models in nonlinear mechanics and examples of this approach applied to a left heart ventricle and to bone tissue may be found in Rodriguez et al. [66].

Chaplain [11] used this inverse approach to analyze an elastic model for mature (non-growing) walls composed of microfibrils embedded in a matrix. The wall is considered to be a hyperelastic incompressible thin membrane with the matrix and the microfibrils represented by separate strain energy functions. The main idea is that the strain energy function for the microfibrils is chosen to provide increased stiffening as the wall deforms. Existing experimental data suggests this to be the case and the physical explanation is that the microfibrils do not come under tension until the wall has experienced some deformation. The strain energy function is decomposed into the strain energy for the matrix material and the strain energy for the fibers as

$$
\Psi=\Psi_{\text {matrix }}+\Psi_{\text {fibers }}
$$

where both strain energies are variants of the Ogden model given by

$$
\Psi=\sum_{k=1}^{n} \mu_{k}\left(\lambda_{1}^{\alpha_{k}}+\lambda_{2}^{\alpha_{k}}+\lambda_{3}^{\alpha_{k}}-3\right) .
$$


Chaplain considered single term $(n=1)$ strain energy functions for both the matrix material and the fibers. The stiffening effect in the fibers was modeled in a phenomenological manner by choosing $\mu_{1}$ and $\alpha_{1}$ to be 'sigmoidal' functions of the principal stretches, i.e. functions that transition from a minimum (relaxed fiber state) to a maximum (stretched fiber state). The strain energy function for the matrix material considers $\mu_{1}$ and $\alpha_{1}$ to be constant parameters.

Spherical and cylindrical walls were considered and the resulting symmetry and thin membrane approximation allows the use of simplified expressions in terms of principal stretches. The internal (turgor) pressure is introduced through mechanical equilibrium and the resulting pressure-extension curves are qualitatively in agreement with experimental data. The phenomenological microfibril model used does not consider the micofibril orientation but this work does show that the stiffening effect of the microfibrils can be modeled in an approach that properly treats material isotropy and frame invariance in a finite deformation setting.

Vandiver and Goriely [75] applied the inverse approach to study the axial growth of cylindrical structures in plants including different strain energy functions in the inner and outer regions of the cylindrical shape. The strain energy functions used were Neo-Hookean strain energy for the inner region

$$
\Psi_{\text {inner }}=\frac{\mu_{\text {inner }}}{2}\left(I_{1}-3\right)
$$

and a Fung type strain energy for a stiffening material

$$
\Psi_{\text {outer }}=\frac{\mu_{\text {outer }}}{2 \nu}\left(e^{\nu\left(I_{1}-3\right)}-1\right),
$$

where $I_{1}$ is the first invariant (the trace) of the Cauchy-Green strain tensor and $\mu$ and $\nu$ are material parameters. A subtle feature of this inverse approach is that since $\mathbf{F}^{G}$ is specified, a constitutive model for $\mathbf{F}^{G}$ is not required. A constitutive model for $\mathbf{F}^{G}$ would be required if the full boundary value problem is to be solved, i.e. if the analyst wishes to prescribe a pressures on a cell wall and determine the resulting deformation and stresses due to growth and elastic response. Development of constitutive models for growth requires experimental data as is succinctly stated in the following phrase from Rodriguez et al. [66] '... the highest priority is the requirement for experimental data to develop an appropriate form of a constitutive law for growth.'

Huang et al. [32] employ the finite deformation formulation described above including a constitutive model for $\mathbf{F}^{G}$ where the wall is composed of a matrix of an isotropic material within which directed microfibrils are embedded. This approach was inspired by the approach used by Gasser et al. [22] in modeling arterial layers. They decompose growth into an isochoric (volume preserving) growth, volumetric expansion and irreversible extension of the microfibrils. The constitutive model is expressed in terms $\mathbf{D}^{G}=\operatorname{sym}\left(\mathbf{L}^{G}\right)$, where $\mathbf{L}^{G}$ is the velocity gradient given in terms of $\mathbf{F}^{G}$ in the intermediate configuration as $\mathbf{L}^{G}=\dot{\mathbf{F}}^{G}\left(\mathbf{F}^{G}\right)^{-1}$. Their constitutive model was obtained using the principle of maximum dissipation [71] in a manner similar to corresponding models for plastic deformation in engineering mechanical behavior, and is expressed in the intermediate frame in terms of rates of effective viscoplastic strains, $\dot{\lambda}$, and overstress functions, $\Phi$,

$$
\mathbf{D}^{G}=\dot{\lambda}_{\mathrm{dev}} \frac{\partial \Phi_{\mathrm{dev}}}{\partial \mathbf{S}}+\dot{\lambda}_{\mathrm{vol}} \frac{\partial \Phi_{\mathrm{vol}}}{\partial \mathbf{S}}+\dot{\lambda}_{\lambda_{a}} \frac{\partial \Phi_{\lambda_{a}}}{\partial \mathbf{S}}
$$

where the first term is the isochoric growth, the second term is the volumetric expansion and the third term is the irreversible extension of the microfibrils. The first term in this constitutive equation may be viewed as a finite deformation generalization of Eq. (3.3) from Dumais et al. [19], while the second term is a model for volumetric expansion that necessarily includes material addition and does not assume a priori 
a constant wall thickness. The third term models the anisotropy due to the presence of microfibrils. In this expression the viscoplastic strains are proportional to the overstress functions and the coefficients of proportionality are material constants that must be provided. The overstress functions are related to the distance of the current stress to the yield function in stress space. The yield functions move due to hardening (and softening) and the hardening models for the isochoric growth and volumetric expansion are arrived at by consideration of new covalent and hydrogen bonds forming new cross-links. The model for irreversible expansion of the microfibrils is a finite deformation extension of the model of Spencer [72]. The inclusion of directed microfibers in a finite deformation framework allows the effects of microfibril reorientation to be represented in the model. The initial microfibril orientation is represented by the unit vector $a_{0}$ and the reoriented microfibril direction is represented by $a$, where $a=\mathbf{F} a_{0}$. Thus the deformation gradient contains the information necessary to follow the reoriented fibers.

The approach used in this work satisfies the continuum mechanics based restriction of frame invariance and allows for the proper treatment of the symmetry properties of the materials composing the cell wall. The constitutive model used may be viewed as a three-dimensional local representation of the MaxwellBingham model that provides the theoretical foundation for the global Augmented Growth Equation, Eq. (2.5). This model includes oriented microfibrils, wall loosening and hardening through the wall thickness, and anisotropic material properties and growth thus representing a significant step forward in the modeling of the mechanics of plant cell wall growth. The authors demonstrate the efficacy of their model in a series of simulations representing cell wall growth by comparing simulation results to experimental results for internode cells of $C$. corallina. These simulations include the elastic deformation of the microfibrils. Some of the simulations reproduced experimental results from Proseus et al. [63] (Fig. 2 in this paper) that have been accurately described by the Augmented Growth Equations. As described earlier, the underlying local constitutive model for the Augmented Growth Equations is the Maxwell-Bingham viscoelastic model. The hyperelastic and rate dependent viscoplastic model used in this work is a three dimensional finite deformation extension of the Maxwell-Bingham viscoelastic model thus it is not surprising that there is good agreement with experimental data that has been accurately described by the Augmented Growth Equations.

\section{System models for growth rate regulation}

Expansive growth rate of pollen tubes have been extensively studied. These single elongating cells exhibit tip growth and oscillatory growth, i.e. the elongation growth rate oscillates in time with a steady amplitude and frequency [12]. Thus these single cells are excellent candidates for investigation in growth rate regulation. It was experimentally observed that cytosolic calcium concentration and secretory vesicle density at the growing tip oscillate at the same frequency as the growth rate, but all are have a different phase $[31,40,58]$. In addition it was shown that inhibition of calcium channels produce a change in growth rate frequency [23]. Recent mathematical models employ a feedback loop to describe the growth rate oscillations observed in pollen tubes.

Kroeger et al. [33,34] constructed models that describe growth rate oscillations of pollen tubes. The predicted results are qualitatively consistent with experimental results. Their models feature a feedback loop that couples the wall mechanical properties (changes in wall viscosity by deposition of new wall material) and wall deformation behavior (irreversible deformation and wall thinning) with the dynamics of biological processes such as stretch-activation of calcium channels and secretion of wall material to the inner wall. The two models can be considered to be system models because coupled equations are provided for the many interrelated mechanical, biological, and chemical processes that are thought to be associated with manipulating the wall's mechanical properties and thus the elongation growth rate. The 
more recent model [34] employs the constitutive equation for a Bingham fluid to obtain a relationship between the strain rate of the wall $(\partial \varepsilon / \partial t)$ and the effective wall stress $\left(\sigma-\sigma_{y}\right)$

$$
\frac{\partial \varepsilon}{\partial t}=F\left(\sigma-\sigma_{y}\right),
$$

where $F$ has the units of the inverse of dynamic viscosity. This constitutive equation replaces Darcy's law that was used in the previous model [33]. The subsequent equations describing the dynamics of the biochemical reactions leading to delivery of wall pectin polymers and changes in wall properties are the same in both models Kroeger et al. [33,34]. The wall stress is related to the turgor pressure by the equilibrium equation, $\sigma=\operatorname{Pr} /(2 h)$, where $r$ is the wall curvature and $h$ is the wall thickness. The strain rate is related to the average elongation growth rate, $V$, by

$$
\frac{\partial \varepsilon}{\partial t}=\frac{\partial v(s)}{\partial s} \approx \frac{V}{S}
$$

where $s$ is the curvilinear coordinate describing the position on the arc beginning at the tube's pole, $v(s)$ is the velocity at each point on the wall arc, and $S$ is the arc length. The rate of change in wall thickness, $\partial h / \partial t$, is modeled as the difference of rate of new wall deposition and the rate of wall thinning due to irreversible deformation (expansion)

$$
\frac{\partial h}{\partial t}=a_{2} C-3 v(s) \frac{r^{2}-r_{i}^{2}}{2 r^{2}},
$$

where wall deposition rate by vesicle secretion (exocytosis) is assumed to be proportional to the cytosolic calcium concentration, $C$, so $a_{2}$ is a constant of proportionality. The rate of change of the calcium concentration near the membrane is described as

$$
\frac{\partial C}{\partial t}=\frac{A}{V_{\mathrm{ol}}}\left(J_{m}-J_{r}\right),
$$

where $A$ is the membrane area, $V_{\mathrm{ol}}$ is the volume of the apex, $J_{m}$ is the calcium ion influx through the calcium channel and $J_{r}$ is the rate by which calcium ion are sequestered by cell organelles within the cytoplasm. The calcium channels are stretch-activated so the conductance is dependent of the membrane stress, $\sigma_{m}$

$$
J_{m}=D_{m}\left(\sigma_{m}\right) \frac{C_{0}-C}{h}
$$

and

$$
D_{m}\left(\sigma_{m}\right)=\frac{D}{1+\exp \left[-a_{3}\left(\sigma_{m}-\sigma_{c}\right)\right]},
$$

where $D$ is the diffusion constant, $a_{3}$ is a constant, and $\sigma_{c}$ is the critical stress. The membrane stress is described in terms of the membrane strain rate (same as the wall strain rate) and dynamic surface viscosity, $\eta$,

$$
\sigma_{m}=\frac{2 \eta}{h_{m}} \frac{\partial \varepsilon}{\partial t} .
$$

The wall extensibility is considered to be the inverse of the wall viscosity, $\mu$. The rate of change in wall viscosity is described as an average of the highly viscous pectin (after de-esterification), $\mu$, and the less viscous pectin that was just secreted to the inner wall, $\mu_{s}$

$$
\frac{\partial \mu}{\partial t}=k\left(\mu_{e q}-\mu\right)-\frac{\mu_{s} a_{2} C}{h},
$$

where $\mu_{e q}$ is the maximum value that is obtained through de-esterification of the pectin monomers. 
The results of the simulations predict oscillations in growth rate, wall thickness, cytoplasmic calcium concentration, and wall viscosity. The predicted phase shift of the oscillation in calcium concentration is approximately $51^{\circ}$ behind the growth rate, near the range obtained experimentally $\left(10^{\circ}-40^{\circ}\right)$. This model was used to evaluate the average growth rate, variation of oscillation period as a function of turgor pressure, and the relationship between secretion rate and the amplitude of the oscillations. Also, the simulations showed that for very short transient turgor pressure changes the instantaneous growth rate is directly proportional to the turgor pressure when the constitutive equation for a Maxwell viscoelastic model is used,

$$
\frac{\partial \varepsilon}{\partial t}=F \sigma+\frac{1}{E} \frac{\partial \sigma}{\partial t}
$$

where $E$ is the elastic modulus. Koeger et al. [34] correctly use the constitutive equation for a Maxwell viscoelastic model to study instantaneous growth rate when the turgor pressure is changing, because temporal changes in turgor pressure will produces temporal changes the wall stresses. This system model may be improved if the constitutive equation for a Bingham Maxwell viscoelastic model, instead of the constitutive equation for a Bingham fluid, were employed for other simulations because the wall stresses are changing with time, even though the turgor pressure is constant. As the simulations demonstrate, wall thickness and wall viscosity both oscillate in time, thus producing temporal changes in the wall stresses. Also, the previous model that employed Darcy's law [33] to study the relationship between the growth rate frequency, extracellular calcium concentration, and calcium channel density within the plasma membrane may be improved by employing the constitutive equation for a Bingham Maxwell viscoelastic model.

Importantly, the authors emphasize the need to conduct more experimental research to validate their models and its many mechanical and biological components. In spite of the need for experimental validation, these models represent an important step forward by modeling the interrelated physical, mechanical, biological, and chemical processes involved in the regulation of growth and growth rate of cells with walls. Perhaps these models will inspire targeted experimental investigations that will lead to experimental validation for individual component models of individual processes and for the overall system model.

Rojas et al. [69] develop a constitutive relationship based on a molecular mechanism that is incorporated into a system model that explores the relationship between the deposition of wall polymers and wall deformation. In this model the wall deformation is considered to be the result of a chemo-rheological process where the effective viscosity of the wall is determined by a chemical reaction. They formulate a mechanism for expansive growth of pollen tubes in which deposition of wall material causes turnover of cross-links between wall polymers and wall deformation is related to the rate of turnover of cross-links. The polymeric network comprising the wall is modeled as a cubic lattice of linear springs representing the cross-links with mesh size $\xi$ and spring constant $\bar{k}$. An expression for the uniaxial stress is

$$
\sigma=\bar{k} \xi \int_{0}^{\infty} \varepsilon \rho(\varepsilon) d \varepsilon=\bar{k} \xi \chi\langle\varepsilon\rangle
$$

where $\chi=\int_{0}^{\infty} \rho(\varepsilon) d \varepsilon$ is the concentration of the cross-links given the probability density of attached springs per unit volume, $\rho(\varepsilon)$, and $\langle\varepsilon\rangle$ is the average elastic strain of the springs. The constitutive model is obtained by setting the time derivative of the uniaxial stress to zero and assuming that the rate of cross-link dissociation per unit volume is $\dot{\chi}=-k_{d} r_{d} \chi$, where $r_{d}$ is the concentration of the de-esterified species and $k_{d}$ is a rate constant. The resulting constitutive model is

$$
\dot{\varepsilon}=k_{d} r_{d}\langle\varepsilon\rangle \text {. }
$$


This model is applied to biaxial stress states by assuming that the strain in direction $i$ may be related to the stresses in the $i$ and $j$ direction through the linear elastic relationship

$$
\left\langle\varepsilon_{i}\right\rangle=\frac{1}{\bar{k} \xi \chi}\left(\sigma_{i}-\nu \sigma_{j}\right)
$$

and this is substituted into the biaxial version of the constitutive model to obtain

$$
\dot{\varepsilon}_{i}=\frac{k_{d} r_{d}}{\bar{k} \xi \chi}\left(\sigma_{i}-\nu \sigma_{j}\right) \text {. }
$$

Equation (4.13) has the desirable property that as $r_{d} \rightarrow 0, \dot{\varepsilon}_{i} \rightarrow 0$ and the material becomes elastic with stresses related to strains by Equation (4.12). Thus model includes both irreversible and elastic deformation. There is a conceptual difficulty with this model development in that the lattice model has zero stress rates while the extension to the biaxial case includes non-zero stress rates. The final form of this constitutive model may be characterized as Newtonian-like fluid with effective viscosity $\mu_{\text {eff }}=\frac{\bar{k} \xi \chi}{k_{d} r_{d}}$ and with the strain and stresses related algebraically through a linear elastic relationship with effective Young's modulus $E_{\text {eff }}=\bar{k} \xi \chi$.

The system model uses geometry similar to Dumais et al. [19] and includes a rate equation for the dynamics of the pectin chemistry in terms of the rate of change of the concentration of the de-esterified species

$$
\dot{r}_{d}=k_{e} r_{e}-2 k_{d} r_{d}\left\langle\varepsilon_{A}\right\rangle-\frac{D}{\delta} r_{d}-v_{s} \frac{\partial r_{d}}{\partial s}
$$

Here $r_{e}$ is the concentration of the esterified species, $k_{e}$ is a rate constant, $\left\langle\varepsilon_{A}\right\rangle$ is the areal strain given by $\left\langle\varepsilon_{A}\right\rangle=\left\langle\varepsilon_{i}\right\rangle+\left\langle\varepsilon_{j}\right\rangle, D$ is the deposition rate, $\delta$ is the wall thickness, $v_{s}$ is the meridional velocity and $s$ is the coordinate in the meridional direction. The first term on the right hand side represents de-esterification, and the next three terms represent cross-linking, deposition and convection. The wall stresses are found from the same equilibrium expressions (given by Equation (3.4)) as in Dumais et al. [19] and the material velocities are related to the strain rates in a manner similar to Dumais et al. [19]. The rate of change of the wall thickness is determined from mass conservation with deposition, mechanical thinning and advection

$$
\dot{\delta}=D-\delta \dot{\varepsilon}_{A}-v_{s} \frac{\partial \delta}{\partial s} .
$$

The model is closed by assuming the deposition rate is regulated through a negative feedback loop of the form

$$
D \sim\left(v_{\text {crit }}-v_{\text {pole }}\right) .
$$

This model reproduces the morphologies of growing pollen tubes. Furthermore, the model predicts growth oscillations that are consistent with experimental results and provides insight to the production of growth oscillations through the mechanism of deposition rate regulation. The molecular model is based on the calcium pectate chemistry model previously proposed by Proseus and Boyer $[7,62]$. The overall system model is a promising step in the analysis of the coupled mechanisms of wall deformation and deposition including wall loosening and the elastic response. The constitutive model is similar to Dumais et al. [19] in that the mechanical aspects of growth are modeled as a flow process with stress related to strain rates but it does not consider anisotropic material behavior as in Dumais et al. [19]. The mechanical constitutive model includes the transition from irreversible deformation in the early stages of growth to elastic deformation in the later stages of growth as the concentration of the de-esterified species $\left(r_{d}\right)$ vanishes. Given this, it may be that future modifications of this model can represent the physics elucidated by in vivo stress relaxation and in vivo creep experiments. 
Other investigators have developed mathematical models that relate calcium dynamics and growth behavior for pollen tubes, and which employ feedback loops $[37,78]$. In all of these models, many assumptions are made and equations employed that must be validated experimentally. In most models, the inclusion and sequencing of the biological processes in the hypothesized system model also need experimental validation.

\section{Conceptual considerations for future models}

All the mathematical models reviewed in this article, with one exception [21], assume that the mechanical properties and mechanical deformation behavior are uniform throughout the thickness of the wall. However, an overview of what is known about the relationships between wall deposition, wall mechanical properties, and elongation growth rate of internode algal cells, pollen tubes, and fungal sporangiophores indicates that this assumption may not accurately represent the growing wall. Prior research indicates that the elongation rates of algal cells, pollen tubes, and fungal cells depend on the magnitude of the mass flow of wall polymers delivered to the inner wall $[3,6,7,12,25,62,77]$. Prior pressure probe experiments $[14,15,47,49,55,63,64]$ demonstrate that magnitude of elongation growth rate depends on (a) the wall's ability to extend irreversibly (related to the magnitude of $\phi$ in Eqs. (2.2) and (2.5)), (b) the area of wall that extends irreversibly (related to the length of the growth zone, $L_{g}$ ), and (c) the magnitude of the effective stress within the wall, (which is related to the magnitude of $P-P_{C}$ in Eqs. (2.2) and (2.5)).

Wall polymers and other wall materials are released to the inner wall surface from the plasma membrane via exocytosis in plant, algal, and fungal cells. So all biological materials that promote wall loosening, irreversible wall deformation, assembly and incorporation of new polymers into the wall, are introduced to the wall at the inner surface only. The outer wall surface is relatively inert with respect to deposition, incorporation, and assembly of new wall materials. The different biological boundary conditions on the inner and outer surfaces of the wall will create a gradient of wall mechanical properties across the wall thickness. The wall near the inner surface will experience more wall loosening and irreversible deformation than the wall near the outer surface. In contrast, the wall near the outer surface will experience more elastic deformation, thus supporting more or most of the wall stresses generated by the turgor pressure.

The magnitude of the gradient of wall mechanical properties and wall deformation behavior will depend on the magnitude of the mass flow of wall polymers and wall loosening agents delivered to the inner wall. To illustrate how different mass flows produce different gradients of mechanical properties and deformation behavior across the wall thickness, consider two sporangiophores growing in the vertical direction, i.e. elongating (Fig. 3). Figure 3 is a schematic illustration of two stage IVb sporangiophores showing the growth zone lengths and cross-sections of the wall within respective growth zones for two cases, when the mass flow of wall materials (wall polymers and wall loosening agents) delivered to the inner cell wall are large (A) and small (B). The wall thickness is the difference in diameter between the outer and inner surface of the wall (Fig. 3, A and B), and is considered to be the same for these two cases. The concentric areas with different shades of green show the hypothesized wall loosening from the inner surface to the outer surface of the wall. The inner most circular area (colored yellow) represents the protoplast of the sporangiophore.

The next concentric area (moving from the inner wall to the outer wall) is very light green in color and represents the wall cross-section where a large amount of wall loosening occurs. It is envisioned that mostly irreversible deformation occurs in this area. This is the area where most stress relaxation occurs, and so new and old wall components can chemically interact in a somewhat low-stress environment. The local constitutive equation that could represent the mechanical behavior of the wall in this area is that of a Bingham fluid. The corresponding global biophysical equation is Eq. (2.2). 
The next concentric area (light green) represents the wall cross-section where there is less wall loosening and cross links between newly synthesized and existing microfibrils are forming and incorporating the new polymers into the wall. It is thought that in this concentric area the wall deformation and intermolecular displacement are beginning to load the matrix cross links that have formed between the new and old wall microfibrils, creating more stresses within the wall. It is envisioned that both irreversible and elastic wall deformations occur in this area, and both $\phi$ and $\varepsilon$ have finite values. The underlying local constitutive equation that could represent the mechanical behavior of the wall this area is that of a Maxwell-Bingham viscoelastic model [45]. The corresponding biophysical global equation is Eq. (2.5).

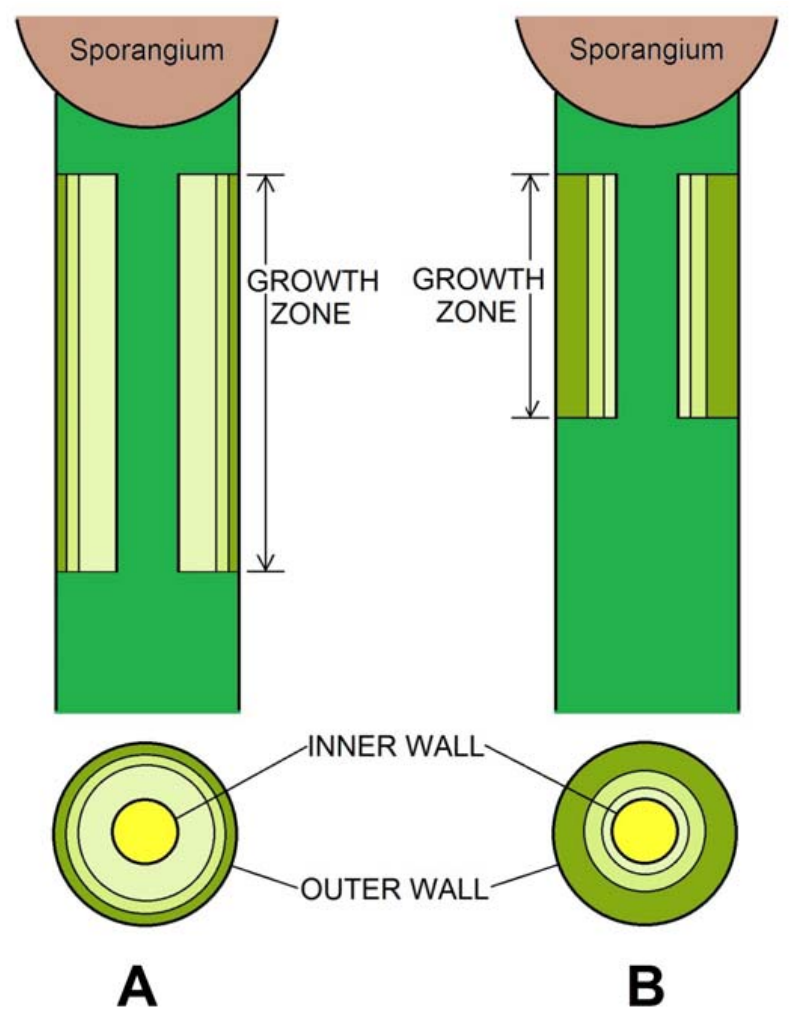

Figure 3. Schematic illustrating the cell wall within the growth zone and between the inner and outer wall surface for two stage IVb sporangiophores when the mass flow of wall-building molecules delivered to the inner cell wall is large (A) and small (B). Reproduced from Ortega (2012) [49], copyrighted by J.K.E. Ortega and is reprinted with permission.

The outer concentric area (darker green) represents the wall cross-section where very little or no wall loosening is occurring. It is thought that the cross-links between microfibils are well formed and load bearing. Most of the elastic deformation within the growth zone occurs in this area and the mechanical properties are predominately characterized by $\varepsilon$. The underlying local constitutive equation that could represent the mechanical behavior of the wall this area is that of an elastic solid. The corresponding biophysical global equation is $\left(d V_{c w c} / d t\right)=(1 / \varepsilon)(d P / d t)$.

It is envisioned that when the mass flow of wall polymers and wall loosening agents delivered to the inner cell wall is large (Fig. $3 \mathrm{~A}$ ), the larger number of transporting vesicles interact with a larger area of 
the plasma membrane and a longer growth zone, $L_{g}$, is produced, compared to the growth zone length when the mass flow of vesicles is smaller (Fig. 3 B). Also, the very light green concentric area adjacent to the protoplast is larger when the mass flow is large (Fig. $3 \mathrm{~A}$ ) compared to the respective area when the mass flow is smaller (Fig. $3 \mathrm{~B}$ ). This implies that the cross-sectional area of wall that exhibits a large amount of wall loosening and irreversible wall deformation (i.e. a large $\phi$ ) is also larger when the mass flow of wall polymers and wall loosening agents is larger, Fig. 3 A compared to Fig. 3 B. The larger magnitudes of $L_{g}$ and $\phi$ will increase the elongation growth rate if the magnitude of the effective turgor pressure, $P-P_{C}$, remains relatively constant. This predicted behavior of the biophysical variables, $L_{g}$, and $\phi$, is consistent with those values measured when elongation growth rate increases [47,49].

In contrast, the outer concentric area (darker green) is smaller for the case when mass flow of wall polymers and wall loosening agents is large (Fig. $3 \mathrm{~A}$ ) compared to the respective area when mass flow rate is small (Fig. 3 B). Because this is the region where the cross links between the new and old microfibrils are load bearing, it is thought that mostly elastic deformation occurs in this region and the elastic properties of this region are characterized by the magnitude of $\varepsilon$. Interestingly, it may be that this outer region is where most of the stress develops that must be exceeded before irreversible deformation can occur and may manifest itself as the magnitude of $P_{C}$. This idea could explain the experimental findings that $\varepsilon$ and $P_{C}$ decreases in magnitude when the elongation rate increases for the sporangiophores of $P$. blakesleeanus [47,49].

In terms of the biophysical variables within the Augmented Growth Equations, this conceptual model predicts that if the mass flow of wall polymers and wall loosening agents delivered to the inner wall surface increases, then the inner concentric area (very light green and representing an region where a large amount of wall loosening occurs) increases, and $L_{g}, \phi$, and elongation rate increase. Concomitantly, the outer concentric area (darker green and representing a region where the cross links between microfibrils are load bearing) decreases, and the magnitudes of $\varepsilon$ and $P_{C}$ decreases. The predicted behaviors of the magnitudes of $L_{g}, \phi, P_{C}, \varepsilon$ and elongation rates are consistent with the measured behaviors of the respective biophysical variables for the sporangiophores of $P$. blakesleeanus $[47,49]$. The conceptual model (Fig. 3) can explain why growing cell walls exhibit both irreversible and elastic deformation and why the biophysical global constitutive equations require terms for both irreversible wall deformation and elastic wall deformation.

The changing mechanical properties along the length of the growth zone of the sporangiophores of P. blakesleeanus [1] can be explained by this conceptual model. Prior research has shown that the longitudinal mechanical extensibility and irreversible deformation is largest in the upper region of the growth zone (nearest the sporangium) for stage IVb sporangiophores and continually decreases toward the lower regions of the growth zone [1]. Below the growth zone the mechanical extensibility is small and all the deformation is elastic [1]. It is hypothesized that most new wall polymers and wall loosening agents are delivered to the inner wall surface in the upper region of the growth zone near the sporangium. The newly added wall polymers 'migrate' down the growth zone because more new wall polymers are added above it as irreversible wall deformation continually thins the wall above it. Concomitantly, the added wall polymers moves toward the outer surface because new wall polymers are laid over it while the wall is deforming and thinning. Thus, the newly added wall polymers move down the growth zone and toward the outer surface as matrix cross-links between microfibrils are being made and becoming load bearing. Now the added wall polymers are cross-linked to microfibrils and undergo elastic deformation as they approach the bottom of the growth zone and the outer surface. This process predicts that the lower end of the growth zone (farthest from the apical tip or sporangium) will be below the lowest region of exocytosis. Furthermore, it is predicted that the lower region of the growth zone will gradually exhibit a smaller amount of irreversible deformation and a larger amount of elastic deformation. The predicted 
behavior of irreversible and elastic deformation along the length of the growth zone is consistent with the mechanical behavior measured experimentally [1].

Elongation growth fluctuation and growth jumps observed during elongation growth of the sporangiophores of P. blakesleeanus can be explained with this conceptual model. During the process described above, if the local stress in a small portion of the wall grows to become exceptionally large (stress concentration) and exceeds the strength of the cross links that are bearing most of the load, those cross links will break causing a sudden dislocation. If the dislocation occurs over a relatively large region it will cause an elongation fluctuation or perhaps even a jump. The dislocation will load other cross links that were not previously loaded and reduce the large local stress by distributing it to surrounding regions i.e. other cross links. It is envisioned that the stress is not uniformly distributed throughout the volume of the cell wall and that large local stresses can build up randomly and somewhat regularly, giving rise to somewhat random and frequent wall dislocations and producing elongation growth fluctuations. Also, it is thought that occasionally the local stress is exceedingly large, causing a large wall dislocation, giving rise to an elongation jump that is occasionally observed during elongation growth. This effect also appears in the model by Dyson et al. [21] as a strain enhanced breakage of longitudinally oriented cross-links between the transversally oriented microfibrils.

Transversely oriented microfibrils embedded in the wall matrix before the dislocation would become more longitudinally oriented during the dislocation. The longitudinal orientation will allow the microfibrils to support more longitudinal loads thus stopping the longitudinal dislocation. This process indicates that the microfibrils will become more longitudinally oriented toward the lower end of the growth zone. This prediction is consistent with fibril reorientation hypothesis previously used to explain left-handed helical growth in stage I and stage IVb sporangiophores of P. blakesleeanus $[50,54]$ and consistent with experimental evidence that indicates the microfibrils are randomly oriented in the transverse direction in the upper regions of the growth zone and more orderly oriented in the longitudinally direction below the growth zone $[10,67,68]$. Analysis of the reorientation of microfibrils embedded in an elastic-plastic material is facilitated by the use of finite deformation continuum models such as that of Huang et al. $[32]$.

The different biological boundary conditions on the inner and outer surfaces of the wall, and the resulting gradient of mechanical properties, will also produce a gradient of stress across the thickness of the wall. It is hypothesized that wall stresses are smallest near the inner surface where wall loosening is largest. Larger stresses develop in regions of the wall where cross-links between microfibrils are load bearing (i.e. the elastic region). Because it is hypothesized that the elastic region is predominately located in the outer portion of the wall thickness, then the "effective thickness", $\delta_{\text {eff }}$, of the wall that supports most of the stresses produced by the turgor pressure is smaller than the measured wall thickness. This implies that the wall stresses are larger than those calculated using the measured wall thickness. Also, this hypothesis predicts that the $\delta_{\text {eff }}$ of the wall will decrease in magnitude as the irreversible wall extensibility and elongation growth rate increase. Furthermore, because the wall stress increase as $\delta_{\text {eff }}$ decreases, it is predicted that the frequency and magnitude of intermolecular dislocation, and fluctuations in elongation growth rate, will be larger for fast growing sporangiophores compared to slow growing sporangiophores. Future experiments will be conducted to evaluate this prediction.

\section{Conclusions and future research}

Models for growth rate, growth rate regulation, and morphogenesis of plant, algal, and fungal cells are beginning to address the many challenges presented by this interdisciplinary and multi-scale problem. In models addressing morphogenesis, computational tools developed in the field of continuum mechanics are 
modified to model the anisotropic mechanical properties and behavior of the wall during expansive growth. In some models that address growth rate regulation, underlying biological and molecular mechanisms obtained from experimental studies are beginning to be incorporated in the models.

Generally, the best mathematical models produce growth behaviors that are consistent with experimental results, explain and provide insight to underlying mechanisms and principles, and predict new phenomena that can be explored experimentally and theoretically. Presently, even though a great deal of progress has been made, the mathematical models are in early stages of development and many problems are yet to be solved in a meaningful way, so that they can accurately model the physical, biological, and chemical processes involved and can make new prediction that can be explored experimentally and theoretically. For example, some of the models that address morphogenesis generate computational artifacts that control morphogenesis and do not relate to physical or biological processes. Some models do not explicitly couple cell wall structure with mechanical properties. Other models do not address wall deposition and assembly in a meaningful way. Many models do not explicitly model irreversible and elastic deformations. Also, many of the equations representing mechanical, biological, and chemical processes in system models addressing growth rate regulation need experimental validation.

Future models of the anisotropic wall properties and anisotropic wall deformation behavior should more explicitly reflect wall architecture and structure. Modeling the associated biological processes together with the wall deformation is just beginning, but this work can provide the foundation for more complete "system models" of expansive growth in the future. Modelling the underlying molecular mechanism(s) of wall deformations could provide valuable insight into both morphogenesis and growth rate regulation, and may suggest new experiments to be conducted for validation purposes and conceptual insight.

This review of mathematical models for expansive growth, morphogenesis, and growth rate regulation of cells with walls highlights the need for more relevant experimental studies to validate new equations used to model individual processes within the system models and to elucidate the mechanisms of individual processes (e.g. wall loosening, wall hardening, polymer incorporation and assembly, etc.). Importantly, more of the mathematical models need to be validated by existing experimental results. It is interesting and important to recognize that of all the models reviewed, only the Augmented Growth Equations (Eqs. (2.4), (2.5), and (2.6)) have reproduced the results presented in Fig. 1 and Fig. 2, and only the model by Huang et al. [32] has reproduced the results presented in Fig. 2. As these models develop it is expected that they will be used to predict new growth behavior and provide important insight into underlying growth processes, as well as reproduce natural growth behavior and growth behavior previously obtained experimentally. In order to achieve these objectives it is important that the models are able to reproduce the experimental results obtained from in vivo stress relaxation experiments (Fig. 1) and the in vivo creep experiments (Fig. 2) to provide evidence that the model obeys the underlying physics measured experimentally. This validation is paramount for all mathematical models of morphogenesis and growth rate regulation if they are to be employed to predict new results to be further explored analytically and experimentally. Eventually all variables and parameters (their magnitudes and behavior) used in the mathematical models must be verified experimentally.

Finally, another area that should warrant additional consideration is modelling the gradient of stress, mechanical properties, and deformation behavior that must exist across the wall thickness. Although the primary wall is thin $(0.1-1.0 \mu \mathrm{m})$, the biological boundary conditions on the inside and outside surfaces are very different. On the inside wall surface adjacent to the plasma membrane there is a constant influx (via exocytosis) of new wall polymers and biologically active molecules that can promote wall loosening, wall hardening, and wall assembly. In addition, microfibrils are synthesized adjacent to the inner wall surface (on the outside surface of the plasma membrane), where they are incorporated and assembled into the inner wall surface along with relevant matrix polymers. New microfibrils and influx of new wall 
materials and biologically active molecules are generally absent on the outer surface of the cell wall. The very different biological boundary conditions on the inner and outer surfaces create a gradient of wall chemical activity that translated to a gradient of wall mechanical properties across the wall thickness. The gradient of wall mechanical properties will result in different deformation behavior and stress distribution across the wall thickness. The conceptual biological model for sporangiophore elongation growth presented in Section 5 (and illustrated in Fig. 3) indicate that significant insight into many features of expansive growth may be obtained by recognizing that the mechanical properties, deformation behavior, and stress distribution are not uniform across the wall thickness. It is apparent that the stress distribution within the wall can, and probably does, change with a change of influx of new wall material, even when the turgor pressure is constant. This requires the use of a local constitutive equation that includes the elastic deformation term even in the cases when the turgor pressure is constant. Future models that account for these different biological boundary conditions on the inner and outer wall surface, and the gradients they produce, may yield new insights that will provide the foundation for better and more insightful mathematical models of expansive growth of cells with walls.

The recent growth models of cells with walls exhibit the potential for significant advancement in the areas of morphogenesis and growth rate regulation. Even though many problems and challenges remain, it is apparent that a great deal of progress has been achieved. These exciting and challenging interdisciplinary and multi-scale problems have attracted investigators from many disciplines and they are bringing many mathematical, physical, biological, and chemical tools to address these problems. It is apparent that the solutions to these many problems and challenges can be facilitated by strong collaborations between investigators coming from diverse disciplines.

Acknowledgements. The authors wish to thank Mariya Ptashnyk for her excellent editorial assistance.

J.K.E.Ortega gratefully acknowledges funding from National Science Foundation Grant MCB-0948921 which supported some of the research reviewed.

\section{References}

[1] C.N. Ahlquist, R.I. Gamow. Phycomyces mechanical behavior of stage II and stage IV. Plant Physiol., 51 (1973), 586-587.

[2] D. Ambrosi, G.A. Ateshian, E.M. Arruda, S.C. Cowin, J. Dumais, A. Goriely, G.A. Holzapfel, J.D. Humphrey, R. Kemkemer, E. Kuhl, J.E. Olberding, L.A. Taber, K. Garikipati. Perspectives on biological growth and remodeling. J Mech. Phys. Solids, 59 (2011), 863-883.

[3] S. Bartnicki-Garcia, C.E. Bracker, G. Glerz, R. Lopez-Franco, H. Lu. Mapping the growth of fungal hyphae orthogonal cell wall expansion during tip growth and the role of turgor. Biophys J., 79 (2000) 2382-2390.

[4] T.I. Baskin. Anisotropic expansion of the plant cell wall. Annu Rev Cell Dev Biol, 21 (2005), 203-222.

[5] E.C. Bingham. Fluidity and Plasticity. McGraw-Hill, New York, 1922.

[6] J. Bove, B. Vaillancourt, J. Kroeger, P.K. Hepler, P.W. Wiseman, A. Geitmann. Magnitude and Direction of Vesicle Dynamics in Growing Pollen Tubes Using Spatiotemporal Image Correlation Spectroscopy and Fluorescence Recovery after Photobleaching. Plant Physiol., 147 (2008), 1646-1658.

[7] J.S. Boyer. Cell wall biosynthesis and the molecular mechanism of plant enlargement. Funct. Plant Biol., 36 (2009), 383-394.

[8] O. Campas, L. Mahadevan. Shape and dynamics of tip-growing cells. Current Biol., 19 (2009), 2102-2107.

[9] N.C. Carpita, D.M. Gibeaut. Structural models of primary cell wall in flowering plants: Consistency of molecular structure with the physical properties of the walls during growth. Plant J., 3 (1993), 1-30.

[10] E.S. Castle. Spiral growth and the reversal of spiraling in Phycomyces, and their bearing on primary wall structure. Am J Botany, 29 (1942), 664-672.

[11] M.A.J. Chaplain. The strain energy function of an ideal plant cell wall. J Theor. Biol., 163 (1993), 77-97.

[12] Y. Chebli, A. Geitmann. Mechanical principles governing pollen tube growth. Funct. Plant Sci Biotech., 1 (2007), $232-245$.

[13] E. Cerda-Olmedo, E.D. Lipson. Phycomyces. Cold Spring Harbor Laboratory Press, Cold Spring Harbor, New York, 1987. 
[14] D.J. Cosgrove. Cell wall yield properties of growing tissue evaluation by in vivo stress relaxation. Plant Physiol., 78 (1985), 347-356.

[15] D.J. Cosgrove. Wall relaxation in growing stems: comparison of four species and assessment of measurement techniques. Planta, 171 (1987), 266-278.

[16] D.J. Cosgrove. Assembly and enlargement of the primary cell wall in plants. Annu. Rev. Cell Dev. Biol., 13 (1997), $171-201$.

[17] D.J. Cosgrove. Loosening of plant cell walls by expansions. Nature, 407 (2000), 321-326.

[18] D.J. Cosgrove. Growth of the plant cell wall. Nat. Rev. Mol. Cell Biol., 6 (2005), 850-861 (doi:10.1038/nrm1746).

[19] J. Dumais, S.L. Shaw, C.R. Steele, S.R. Long, P.M. Ray. An anisotropic-viscoplastic model of plant cell morphogenesis by tip growth. Int. J Dev. Biol., 50 (2006), 209-222.

[20] R.J. Dyson, O.E. Jensen. A fibre-reinforced fluid model of anisotropic plant cell growth. J Fluid Mech., 655 (2010), 472-503.

[21] R.J. Dyson, L.R. Band, O.E. Jensen. A model of cross-link kinetics in the expanding plant cell wall: Yield stress and enzyme action. J Theor Biol., 307 (2012), 125-136.

[22] T.C. Gasser, R.W. Ogden, G.A. Holzapfel. Hyperelastic modelling of arterial layers with distributed collagen fibre orientations. J Roy Soc. Interface, 3 (2006), 15-35.

[23] A. Geitmann, Y.Q. Li, M. Cresti. The role of the cytoskeleton and dyctyosome activity in the pulsatory growth of Nicotiana tabacum and Petunia hybrida. Bot. Acta, 109 (1996), 102-109.

[24] A. Geitmann, J.K.E. Ortega. Mechanics and modeling of plant cell growth. Trends Plant Sci., 14 (2009), $467-478$.

[25] G. Gierz, S. Bartnicki-Garcia. A three-dimensional model of fungal morphogenesis based on the vesicle supply center concept. J Theor. Biol., 208 (2001), 151-164.

[26] J.E.F. Green, A. Friedman. The extensional flow of a thin sheet of incompressible, transversly isotropic fluid. Europ. J Appl. Math., 3 (2008), 225-257.

[27] P.B. Green. Growth Physics in Nitella: a Method for Continuous in Vivo Analysis of Extensibility Based on a Micromanometer Technique for Turgor Pressure. Plant Physiol., 43 (1968), 1169-1184.

[28] P.B. Green. Cell Morphogenesis. Ann. Rev. Plant Physiol., 20 (1969), 365-394.

[29] P.B. Green, R.O. Erickson, J. Buggy. Metabolic and physical control of cell elongation rate: in vivo studies in Nitella. Plant Physiol., 47 (1971), 423-430.

[30] I.B. Heath. Tip growth in plant and fungal cells. Academic Press, Inc., San Diego, CA , 1990.

[31] T. Holdaway-Clarke, P. Hepler. Control of pollen tube growth: role of ion gradients and fluxes. New Phytol., 159 (2003), 539-563.

[32] R. Huang, A.A. Becker, I.A. Jones. Modelling cell wall growth using a fibre-reinforced hyperelastic-viscoplastic constitutive law. J Mech. Phys. Solids, 60 (2012), 750-783.

[33] J.H. Kroeger, A. Geitmann, M. Grant. Model for calcium dependent oscillatory growth in pollen tubes. J Theor. Biol., 253 (2008), 363-374.

[34] J.H. Kroeger, R. Zerzour, A. Geitmann. Regulator or driving force? The role of turgor pressure in oscillatory plant cell growth. PLoS One, 6 (2011), e18549.

[35] J.H. Kroeger, A. Geitmann. Pollen tube growth: Getting a grip on cell biology through modeling. Mech. Res. Comm., 42 (2012), 32-39.

[36] S. Lewicka. General and analytic solutions of the Ortega equation. Plant Physiol., 142 (2006), 1493-1510.

[37] J. Liu, B.M.A.G. Piette, M.J. Deeks, V.E. Franklin-Tong, P.J. Hussey. A compartmental model analysis of integrative and self-regulatory ion dynamics in pollen tube growth Plos One, 5 (2010), e13157.

[38] J.A. Lockhart. An analysis of irreversible plant cell elongation J Theor. Biol., 8 (1965), 264-275.

[39] F. Marga, M. Grandbois, D.J. Cosgrove, T.I. Baskin. Cell wall extension results in the coordinate separation of parallel microfibrils: evidence from scanning electron microscopy and atomic force microscopy. Plant J., 43 (2005), $181-190$.

[40] M.A. Messerli, R. Greton, L.F. Jaffe, K.R. Robinson. Periodic increases in elongation rate precede increases in cytosolic Ca2+ during pollen tube growth. Dev. Biol., 222 (2000), 84-98.

[41] F.J. Molz, J.S. Boyer. Growth-induced water potential in plant cells and tissue. Plant Physiol., 62 (1978), $423-429$.

[42] R. Murphy, J.K.E. Ortega. A new pressure probe method to determine the average volumetric elastic modulus of cells in plant tissue. Plant Physiol., 107 (1995), 995-1005.

[43] R. Murphy, J.K.E. Ortega. A study of the stationary volumetric elastic modulus during dehydration and rehydration of stems of pea seedlings. Plant Physiol., 110 (1996), 1309-1316.

[44] H. Nonami, J.S. Boyer. Direct demonstration of a growth-induced water potential gradient. Plant Physiol., 102 (1993), $13-19$.

[45] J.K.E. Ortega. Augmented growth equation for cell wall expansion. Plant Physiol., 79 (1985), 318-320.

[46] J.K.E. Ortega. Governing equations for plant cell growth. Physiol. Plant, 79 (1990), 116-121.

[47] J.K.E. Ortega. A quantitative biophysical perspective of expansive growth for cells with walls. Ed. SG Pandalai, Rec. Res. Dev. Biophys, Transworld Research Network, Kerala, India. 3 (2004), 297-324. 
[48] J.K.E. Ortega. Plant cell growth in tissue. Plant Physiol., 154 (2010), 1244-1253.

[49] J.K.E. Ortega. Growth rate regulation of cells with walls: The sporangiophores of Phycomyces blakesleeanus used as a model system. Rec. Res. Dev. Plant Physiol., 5 (2012), 1-19.

[50] J.K.E. Ortega, R.I. Gamow. The problem of handedness reversal during the spiral growth of Phycomyces. J Theor. Biol., 47 (1974), 317-332.

[51] J.K.E. Ortega, R.G. Keanini, K.J. Manica. Pressure probe technique to study transpiration in Phycomyces sporangiophores. Plant Physiol., 87 (1988), 11-14.

[52] J.K.E. Ortega, K.J. Manica, R.G. Keanini. Phycomyces: Turgor pressure behavior during the light and avoidance growth response. Photochem. Photobiol., 48 (1988), 697-703.

[53] J.K.E. Ortega, E.G. Zehr, R.G. Keanini. In vivo creep and stress relaxation experiments to determine the wall extensibility and yield threshold for the sporangiophores of Phycomyces. Biophys. J., 56 (1989), 465-475.

[54] J.K.E. Ortega, G.E. Lesh-Laurie, M.A. Espinosa, E.L. Ortega, S.M. Manos, M.D. Cunning, J.E.C. Olson. Helical growth of stage-IVb sporangiophores Phycomyces blakesleeanus: the relationship between rotation and elongation growth rates. Planta, 216 (2003), 716-722.

[55] J.K.E. Ortega, C.M. Munoz, S.E. Blakley, J.T. Truong, E.L. Ortega. Stiff mutant genes of Phycomyces affect turgor pressure and wall mechanical properties to regulate elongation growth rate. Frontiers in Plant Science, 3 (2012), 1-12.

[56] J.K.E. Ortega, M.E. Smith, A.J. Erazo, M.A. Espinosa, S.A. Bell, E.G. Zehr. A comparison of cell-wall-yielding properties for two developmental stages of Phycomyces sporangiophores: Determination by in-vivo creep experiments. Planta, 183 (1991), 613-619.

[57] E. Parre, A. Geitmann. Pectin and the role of the physical properties of the cell wall in pollen tube growth of Solanum chacoense. Planta, 220 (2005), 582-592.

[58] R. Parton, S. Fischer-Parton, M. Watahiki, A. Trewavas. Dynamics of the apical vesicle accumulation and the rate of growth are related in individual pollen tubes J Cell Sci., 114 (2001), 2685-2695.

[59] J.B. Passioura, S.C. Fry. Turgor and cell expansion: beyond the Lockhart equation. Austral. J Plant Physiol., 19 (1992), 565-576.

[60] M. Pietruszka. Solutions for a local equation of anisotropic plant cell growth: an analytical study of expansin activity. J Royal Soc. Int., 8 (2011), 975-987.

[61] M. Pietruszka. Special solutions to the Ortega Equation. J Plant Growth Regul., 32 (2013), 102-107.

[62] T.E. Proseus, J.S. Boyer. Calcium deprivation disrupts enlargement of Chara corallina cells: further evidence for the calcium pectate cycle. J Exp. Bot., 63 (2012), 3953-3958.

[63] T.E. Proseus, J.K.E. Ortega, J.S. Boyer. Separating growth from elastic deformation during cell enlargement. Plant Physiol., 119 (1999), 775-784.

[64] T.E. Proseus, G.L. Zhu, J.S. Boyer. Turgor, temperature and the growth of plant cells:using Chara corallina as a model system. J. Exp. Bot., 51 (2000), 1481-1494.

[65] P.A. Richmond, J.-P. Métraux, L. Taiz. Cell expansion patterns and directionality of wall mechanical properties in Nitella Plant Physiol., 65 (1980), 211-217.

[66] E.K. Rodriguez, A. Hoger, A. McCulloch. Stress-dependent finite growth in soft elastic tissue. J. Biomechanics, 27 (1994), 455-467.

[67] P.A. Roelofsen. The origin of spiral growth in Phycomyces sporangiophores. Record of Travaux Botaniques Neerlandais, 42 (1950), 72-110.

[68] P. Roelofsen. Cell wall structure in the growth zone of Phycomyces sporangiophores. II. Double refraction and electron microscopy. The origin of spiral growth in Phycomyces sporangiophores. Biochemica et Biophysica Acta, 6 (1951), 357-373.

[69] E.R. Rojas, S. Hotton, J. Dumais. Chemically-mediated Mechanical expansion of the pollen tube cell wall. Biophys. J., 101 (2011), 1844-1853.

[70] J. Ruiz-Herrera. Fungal cell wall: Structure, synthesis, and assembly. CRC Press, New York, 2012.

[71] J.C. Simo, T.J.R. Hughes. Computational Inelasticity. Springer, New York, 1998.

[72] A.J.M. Spencer. A theory of viscoplasticity for fabric-reinforced composites. J Mech. Phys. Solids, 49 (2001), $2667-2687$.

[73] L. Taiz. Plant Cell Expansion: Regulation of Cell Wall Mechanical Properties. Ann. Rev. Plant Physiol., 35 (1984), $585-657$.

[74] A.-C. Tang, J.S. Boyer. Xylem tension affect growth-induced water potential and daily elongation of maize leaves. J Exp. Bot., 59 (2008), 753-764.

[75] R. Vandiver, A. Goriely. Tissue tension and axial growth of cylindrical structures in plants and elastic tissues. Europhys. Letter, 84 (2008), 58004.

[76] B. Veytsmann, D.J. Cosgrove. A model of cell wall expansion based on thermodynamics of polymer networks. Biophys. J., 75 (1998), 2240-2250.

[77] J.G.H. Wessel. Tip growth in plant and fungal cells. IB Heath (Ed.), Academic Press, Inc., San Diego, CA (1990), 1-29.

[78] A. Yan, G. Xu, Z.-B.Yang. Calcium participates in feedback regulation of the oscillating ROP1 Rho GTPase in pollen tubes. PNAS, 106 (2009), 22002-22007. 